\title{
The Heat Semigroup and Integrability of Lie Algebras: Lipschitz Spaces and Smoothness Properties
}

\author{
Derek W. Robinson \\ Mathematics Research Section, School of Mathematical Sciences, \\ Australian National University, Canberra
}

\section{Dedicated to Res Jost and Arthur Wightman}

\begin{abstract}
We define and analyze Lipschitz spaces $\mathscr{B}_{\alpha, q}$ associated with a representation $x \in g \rightarrow V(x)$ of the Lie algebra $g$ by closed operators $V(x)$ on the Banach space $\mathscr{B}$ together with a heat semigroup $S$. If the action of $S$ satisfies certain minimal smoothness hypotheses with respect to the differential structure of $(\mathscr{B}, g, V)$ then the Lipschitz spaces support representations of $g$ for which products $V(x) V(y)$ are relatively bounded by the Laplacian generating $S$. These regularity properties of the $\mathscr{B}_{\alpha, q}$ can then be exploited to obtain improved smoothness properties of $S$ on $\mathscr{B}$. In particular $C_{4}$-estimates on the action of $S$ automatically imply $C_{\infty}$-estimates. Finally we use these results to discuss integrability criteria for $(\mathscr{B}, g, V)$.
\end{abstract}

\section{Introduction}

Let $(\mathscr{B}, g, V)$ be a representation of the Lie algebra $g$ by a family of closable operators $V=\{V(x) ; x \in g\}$ acting on a dense invariant subspace $\mathscr{B}_{\infty}$ of the Banach space $\mathscr{B}$ and let

$$
\Delta=-\sum_{i=1}^{d} V\left(x_{i}\right)^{2}
$$

denote the Laplacian associated with the basis $x_{1}, \ldots, x_{d}$ of $g$. If the $V(x)$ satisfy the usual dissipation properties required for generators of continuous one-parameter groups then it follows from [BGJR] that $\left(\mathscr{B}_{\infty}, g, V\right)$ integrates to a continuous representation $U$ of the corresponding connected Lie group $G$ if, and only if, $\Delta$ is closable and its closure $\bar{\Delta}$ generates a continuous semigroup $S$ satisfying certain smoothness properties. These latter properties are of two kinds; range conditions $S_{t} \mathscr{B} \subseteq \mathscr{B}_{n}$, where $\mathscr{B}_{n}$ is the common domain of all $n$th order monomials in the $V(x)_{j}$, and boundedness conditions

$$
\left\|V\left(x_{i_{1}}\right) \ldots V\left(x_{i_{n}}\right) S_{t}\right\|=\mathrm{O}\left(t^{-n / 2}\right)
$$


as $t \rightarrow 0$. Specifically Theorem 2.10 of [BGJR] proves integrability if $S$ satisfies the range conditions for all $n \geqq 1$. The principle aim of this paper is to strengthen this result by weakening the range assumption.

The key step in the integrability result of [BGJR] is the proof that the boundedness condition for $n=1$ combined with the range condition for all $n \geqq 1$ implies the boundedness condition for all $n \geqq 1$. But in Theorem 6.1 we establish the same conclusion from the assumption that the range condition is satisfied for $n=4$. Therefore the integrability theorem of [BGJR] can be considerably extended. It suffices that the heat semigroup $S$ satisfies $C_{4}$-smoothness properties instead of $C_{\infty}$ properties. In the special case of unitary representations Nelson's integrability result [Nes] is based on $C_{3}$-conditions but the Banach space theorem uses an interpolation argument which introduces the stronger $C_{4}$-requirement.

Our discussion of smoothness conditions indeed follows a very indirect path. We use the minimal smoothness conditions to construct Lipschitz spaces which interpolate between $\mathscr{B}$ and $\mathscr{B}_{1}$. These spaces support representations of $g$ with greater regularity properties which ensure that the heat semigroup acts in a smoother fashion on the Lipschitz spaces. Hence its action on $\mathscr{B}$ is also smooth. Unfortunately the discussion of the Lipschitz spaces requires some intricate analysis to obtain equivalent characterizations which emphasize distinct features of the spaces. This type of analysis is commonplace for the classical Lipschitz spaces and appears to be an unavoidable part of the present approach.

\section{General Formalism}

In this section we introduce the basic definitions and notation for representations of Lie algebras. Then we recall some basic facts about Lipschitz spaces associated with semigroups and, in particular, with holomorphic semigroups.

\section{a) Lie Algebras}

First, if $W$ denotes a family of norm-closable operators on a Banach space $\mathscr{B}$ then we define the $C_{n}$-elements $\mathscr{B}_{n}\left(=\mathscr{B}_{n}(W)\right)$ by

$$
\mathscr{B}_{n}=\bigcap_{W_{1}, \ldots, W_{n} \in W} D\left(\bar{W}_{1} \ldots \bar{W}_{n}\right)
$$

and the $C_{\infty}$-elements $\mathscr{B}_{\infty}\left(=\mathscr{B}_{\infty}(W)\right)$ by

$$
\mathscr{B}_{\infty}=\bigcap_{n \geqq 1} \mathscr{B}_{n}
$$

Second, if $g$ is a Lie algebra we define a representation $(\mathscr{B}, g, V)$ to be a family of closed operators $V=\{V(x) ; x \in g\}$ on $\mathscr{B}$ such that

1. $\mathscr{B}_{\infty}(V)$ is norm-dense

2. $V(x+y) a=V(x) a+V(y) a, \quad a \in \mathscr{B}_{1}, x, y \in g$,

3. $(\operatorname{ad} V(x))(V(y)) a=V((\operatorname{ad} x)(y)) a, \quad a \in \mathscr{B}_{2}, x, y \in g$. 
Third, if $x_{1}, \ldots, x_{d}$ is a basis of $g$ we define seminorms $\varrho_{n}$, and norms $\|\cdot\|_{n}, n \geqq 1$ by

$$
\begin{gathered}
\varrho_{n}(a)=\sup _{1 \leqq i_{1}, \ldots, i_{n} \leqq d}\left\|V\left(x_{i}\right) \ldots V\left(x_{i_{n}}\right) a\right\| \\
\|a\|_{n}=\|a\|+\sup _{1 \leqq m \leqq n} \varrho_{m}(a)
\end{gathered}
$$

for $a \in \mathscr{B}_{n}(V)$. Then $\mathscr{B}_{n}(V)$ is a Banach space with respect to the norm $\|\cdot\|_{n}$ and $\mathscr{B}_{\infty}(V)$ is a Fréchet space with respect to the topology defined by the family of norms $\left\{\|\cdot\|_{n} ; n \geqq 1\right\}$. In addition one has $\mathscr{B} \supseteq \mathscr{B}_{1} \supseteq \mathscr{B}_{2} \supseteq \ldots \supseteq \mathscr{B}_{n} \supseteq \ldots$, where the inclusion sign denotes a continuous embedding of Banach spaces. Note that in the sequel the inclusion sign always has this interpretation. In particular the equality of the Banach spaces is understood to incorporate equivalence of norms.

Next we associate with each basis $x_{1}, \ldots, x_{d}$ of $g$ a Laplacian

In particular

$$
\Delta=-\sum_{i=1}^{d} V\left(x_{i}\right)^{2} \text {. }
$$

$$
D(\Delta)=\bigcap_{i=1}^{d} D\left(V\left(x_{i}\right)^{2}\right) \supseteq \mathscr{B}_{2} .
$$

If $\Delta$ has a norm-closed extension $\hat{\Delta}$ which generates a continuous semigroup $S$ then $S$ is called a heat semigroup. In the sequel we assume the existence of a heat semigroup with an action which is "smooth" relative to the differential structure $\left(\mathscr{B}_{n},\|\cdot\|_{n}\right)$ associated with the representation. Typically we assume that $S_{t} \mathscr{B} \subseteq \mathscr{B}_{2}$ for $t>0$. But this condition automatically implies that $\mathscr{B}_{2}$ is a core of $\hat{\Delta}$. Therefore $\hat{\Delta}=\bar{\Delta}$ the closure of $\Delta$.

\section{b) Lipschitz Spaces}

Let $T$ denote a strongly continuous semigroup on the Banach space $\mathscr{B}$ and $\alpha, q$ real parameters with values $\alpha \in\langle 0,1\rangle, q \in[1, \infty]$, or $\alpha=1, q=\infty$. The Lipschitz space $\mathscr{B}_{\alpha, q}^{T}$ is then defined by

$$
\mathscr{B}_{\alpha, q}^{T}=\left\{a \in \mathscr{B} ; t \rightarrow t^{-\alpha}\left\|\left(I-T_{t}\right) a\right\| \in L_{q}(d \mu ;\langle 0,1\rangle)\right\},
$$

where $d \mu(t)=d t / t$. It follows that $\mathscr{B}_{\alpha, q}^{T}$ is a Banach space with respect to the norm

$$
q \rightarrow\|a\|_{\alpha, q}^{T}=\|a\|+\left(\int_{0}^{1} d \mu(t)\left(t^{-\alpha}\left\|\left(I-T_{t}\right) a\right\|\right)^{q}\right)^{1 / q}
$$

if $q<\infty$, or the corresponding supremum norm if $q=\infty$. (For details on Lipschitz spaces associated with semigroups we refer to the books by Butzer and Berens [BuB] and Triebel [Tri].) It follows that $\mathscr{B}_{\alpha, q}^{T}$ is a norm-dense $T$-invariant subspace of $\mathscr{B}$. Moreover, if $H$ denotes the generator of $T$ then $D(H) \subseteq \mathscr{B}_{\alpha, q}^{T}$ and the spaces $\mathscr{B}_{\alpha, q}^{T}$ interpolate between $\mathscr{B}$ and the subspace $D(H)$ equipped with the graph norm.

Since $T$ is continuous there exist $N \geqq 1$ and $\varrho \geqq 0$ such that $\left\|T_{t}\right\| \leqq N \exp \{\varrho t\}$, $t \geqq 0$. Therefore $H_{\varrho}=H+\varrho I$ generates a continuous semigroup $T^{\varrho}$ which satisfies the uniform bound $\left\|T_{t}^{\varrho}\right\| \leqq N, t \geqq 0$. This change does not affect the Lipschitz spaces. 
One has

(with equivalent norms).

$$
\mathscr{B}_{\alpha, q}^{T}=\mathscr{B}_{\alpha q}^{T^{e}}
$$

Next if $T$ is holomorphic there are a variety of equivalent characterizations of the Lipschitz spaces. Holomorphy is equivalent to the statement that $T_{t} \mathscr{B} \subseteq D(H)$ for $t>0$ and there is a $c>0$ such that $\left\|H T_{t}\right\| \leqq c t^{-1}$ for $t \in\langle 0,1\rangle$. Then by a slight variation of a theorem of Peetre ([BuB] Theorem 3.5.3)

$$
\mathscr{B}_{\alpha, q}^{T}=\left\{a \in \mathscr{B} ; t \rightarrow t^{m-\alpha}\left\|H^{m} T_{t} a\right\| \in L_{q}(d \mu ;\langle 0,1\rangle)\right\}
$$

for $m=1,2, \ldots$, and $\|\cdot\|_{\alpha, q}^{T}$ is equivalent to the norms

$$
a \rightarrow\|a\|+\left(\int_{0}^{1} d \mu(t)\left(t^{m-\alpha}\left\|H^{m} T_{t} a\right\|\right)^{q}\right)^{1 / q}
$$

if $q<\infty$ with the obvious modification if $q=\infty$. Alternatively

$$
\mathscr{B}_{\alpha, q}^{T}=\left\{a \in \mathscr{B} ; t \rightarrow t^{m-\alpha}\left\|\left(I+H_{\varrho}\right)^{m} T_{t}^{o} a\right\| e^{-t} \in L_{q}(d \mu ;\langle 0, \infty\rangle\}\right.
$$

for $m=1,2, \ldots$, and $\|\cdot\|_{\alpha, q}^{T}$ is equivalent to the norms

$$
\begin{gathered}
a \rightarrow\left(\int_{0}^{1} d \mu(t)\left(t^{m-\alpha}\left\|\left(I+H_{\varrho}\right)^{m} T_{t} a\right\|\right)^{q}\right)^{1 / q}, \\
a \rightarrow\left(\int_{0}^{\infty} d \mu(t)\left(t^{m-\alpha}\left\|\left(I+H_{\varrho}\right)^{m} T_{t}^{\varrho} a\right\| e^{-t}\right)^{q}\right)^{1 / q}
\end{gathered}
$$

if $q<\infty$, and the supremum norms if $q=\infty$. Basically these last two equivalences follow from applying Peetre's theorem to the semigroup with generator $\left(I+H_{\varrho}\right)$, i.e. the semigroup $t \rightarrow T_{t} \exp \{-(1+\varrho) t\}=T_{t}^{\varrho} \exp \{-t\}$. But there is one new feature. The functionals $\varrho_{\alpha, q ; m}$ defined by (2.8) are norms which dominate the norm $\|\cdot\|$.

Clearly the $\varrho_{\alpha, q ; m}$ are seminorms but if $\varrho_{\alpha, q ; m}(a)=0$ then $\left\|\left(I+H_{\varrho}\right)^{m} T_{t} a\right\|=0$ for all $t \in\langle 0,1\rangle$. Now the usual resolvent estimates give the dissipativity condition

$$
\left\|\left(I+H_{\varrho}\right)^{m} b\right\| \geqq N^{-1}\|b\|
$$

for all $b \in D\left(H^{m}\right)$. Hence $\varrho_{\alpha, q ; m}(a)=0$ implies $\left\|T_{t} a\right\|=0$ for $t \in\langle 0,1\rangle$ and by continuity $\|a\|=0$. Thus $\varrho_{\alpha, q ; m}$ is a norm. Next let $a_{n} \in \mathscr{B}_{\alpha, q ; m}$, where

$$
\mathscr{B}_{\alpha, q ; m}=\left\{a \in \mathscr{B} ; t \rightarrow t^{m-\alpha}\left\|\left(I+H_{\varrho}\right)^{m} T_{t} a\right\| \in L_{q}(d \mu ;\langle 0,1\rangle)\right\}
$$

and suppose $\varrho_{\alpha, q ; m}\left(a_{n}\right) \rightarrow 0$ as $n \rightarrow \infty$ and $\left\|a_{n}-a\right\| \rightarrow 0$ for some $a \in \mathscr{B}$. Then

$$
\begin{aligned}
\left(\int_{\varepsilon}^{1} d \mu(t)\left(t^{m-\alpha}\left\|\left(I+H_{\varrho}\right)^{m} T_{t} a\right\|\right)^{q}\right)^{1 / q} & \leqq \lim _{n \rightarrow \infty}\left(\int_{\varepsilon}^{1} d \mu(t)\left(t^{m-\alpha}\left\|\left(I+H_{\varrho}\right)^{m} T_{t}\left(a-a_{n}\right)\right\|\right)^{q}\right)^{1 / q} \\
& \leqq \lim _{n \rightarrow \infty} c\left\|a-a_{n}\right\|=0
\end{aligned}
$$

where the second estimate follows from the holomorphy of $T$. Therefore $a \in \mathscr{B}_{\alpha, q ; m}$ and $\varrho_{\alpha, q ; m}(a)=0$. Hence the identity map from $\mathscr{B}_{\alpha, q ; m}$ into $\mathscr{B}$ is closed. Consequently, by the closed graph theorem, there exist $C_{\alpha, q ; m}>0$ such that $\|a\| \leqq C_{\alpha, q ; m} \varrho_{\alpha, q ; m}(a)$ for all $a \in \mathscr{B}_{\alpha, q ; m}$. Similarly the functionals given by (2.9) are norms which dominate $\|\cdot\|$. 
Next we consider the Poisson semigroup associated with a uniformly bounded semigroup and the corresponding Lipschitz spaces.

Assume now that the continuous semigroup $T$, with generator $H$, satisfies the uniform bound $\left\|T_{t}\right\| \leqq N, t \geqq 0$. The Poisson semigroup $P$ associated with $T$ is the semigroup generated by $H^{1 / 2}$. It is given in terms of $T$ by the algorithm

$$
P_{t}=(4 \pi)^{-1 / 2} t \int_{0}^{\infty} d s v_{t}(s) T_{s}
$$

where $v_{t}(s)=s^{3 / 2} \exp \left\{-t^{2} / 4 s\right\}$. It follows that $\left\|P_{t}\right\| \leqq N$ and $P$ is automatically holomorphic (see, for example, [BeF] or [Yos] for details).

If $\mathscr{B}_{\alpha, q}^{P}$ are the Lipschitz spaces corresponding to $P$ then one has the identification

$$
\mathscr{B}_{\alpha, q}^{P}=\mathscr{B}_{\alpha / 2, q}^{T} .
$$

Moreover, since $P$ is holomorphic $\mathscr{B}_{\alpha, q}^{P}$ has the alternative characterizations

$$
\begin{aligned}
& \mathscr{B}_{\alpha, q}^{P}=\left\{a \in \mathscr{B} ; t \rightarrow t^{m-\alpha}\left\|H^{m / 2} P_{t} a\right\| \in L_{q}(d \mu ;\langle 0,1\rangle)\right\}, \\
& \mathscr{B}_{\alpha, q}^{P}=\left\{a \in \mathscr{B} ; t \rightarrow t^{m-\alpha}\left\|\left(I+H^{1 / 2}\right)^{m} P_{t} a\right\| e^{-t} \in L_{q}(d \mu ;\langle 0, \infty\rangle)\right\},
\end{aligned}
$$

and $\|\cdot\|_{\alpha, q}^{P}$ is equivalent to the norms

$$
\begin{aligned}
& a \rightarrow\|a\|+\left(\int_{0}^{1} d \mu(t)\left(t^{m-\alpha}\left\|H^{m / 2} P_{t} a\right\|\right)^{q}\right)^{1 / q} \\
& a \rightarrow\left(\int_{0}^{1} d \mu(t)\left(t^{m-\alpha}\left\|\left(I+H^{1 / 2}\right)^{m} P_{t} a\right\|\right)^{q}\right)^{1 / q} \\
& a \rightarrow\left(\int_{0}^{\infty} d \mu(t)\left(t^{m-\alpha}\left\|\left(I+H^{1 / 2}\right)^{m} P_{t} a\right\| e^{-t}\right)^{q}\right)^{1 / q} .
\end{aligned}
$$

This follows from (2.5)-(2.9) with $P$ replacing $T$ or $T^{\varrho}$ and $H^{1 / 2}$ replacing $H$ or $H_{\varrho}$.

Finally let $Q$ denote the Poisson semigroup associated with the modified semigroup $T \rightarrow R_{t}=T_{t} e^{-t}$ with generator $I+H$. Since $\mathscr{B}_{\alpha, q}^{T}$ is unchanged by the replacement $T \rightarrow R$ the space $\mathscr{B}_{\alpha, q}^{\boldsymbol{P}}$ is unchanged by the replacement $P \rightarrow Q$. Hence

$$
\mathscr{B}_{\alpha, q}^{P}=\left\{a \in \mathscr{B} ; t \rightarrow t^{m-\alpha}\left\|(I+H)^{m / 2} Q_{t} a\right\| \in L_{q}(d \mu ;\langle 0,1\rangle)\right\}
$$

and $\|\cdot\|_{\alpha, q}^{\boldsymbol{P}}$ is equivalent to the norms

$$
a \rightarrow\|a\|+\left(\int_{0}^{1} d \mu(t)\left(t^{m-\alpha}\left\|(I+H)^{m / 2} Q_{t} a\right\|\right)^{q}\right)^{1 / q}
$$

by (2.15). But it follows from the closed graph theorem that the second term in this expression is a norm which dominates the norm $\|\cdot\|$. This is deduced by a similar argument used for (2.9) above. Therefore $\|\cdot\|_{\alpha, q}^{\boldsymbol{P}}$ is equivalent to the norms

$$
a \rightarrow\left(\int_{0}^{1} d \mu(t)\left(t^{m-\alpha}\left\|(I+H)^{m / 2} Q_{t} a\right\|\right)^{q}\right)^{1 / q} .
$$




\section{The Heat Semigroup}

In this section we consider a heat semigroup $S$ associated with the representation $(\mathscr{B}, g, V)$ and analyze the implications of smoothness conditions on its action relative to the differential structure $\left(\mathscr{B}_{n},\|\cdot\|_{n}\right)$. Subsequently we use the results of this analysis to characterize the Lipschitz spaces $\mathscr{B}_{\alpha, q}^{S}$ in terms of the differential structure under minimal smoothness hypotheses.

\section{a) Smoothness Conditions}

We assume in the sequel the existence of a strongly continuous heat semigroup $S$. Continuity automatically implies bounds $\left\|S_{t}\right\| \leqq N \exp \{\varrho t\}$ for all $t \geqq 0$ with $N \geqq 1$ and $\varrho \geqq 0$ and the pair $(N, \varrho)$ will subsequently always indicate such bounds.

We consider two types of regularity condition on $S$, range restrictions and local boundedness.

Condition $S_{n}$. For each $t>0$ the heat semigroup $S$ satisfies $S_{t} \mathscr{B} \subseteq \mathscr{B}_{n}$.

If this condition is satisfied for $n \geqq 2$ then $\mathscr{B}_{n}$ is a core of $\hat{\Delta}$ and one has $\hat{\Delta}=\bar{\Delta}$. Alternatively if Condition $S_{1}$ is satisfied then $\mathscr{B}_{1} \cap D(\hat{\Delta})$ is a core of $\hat{\Delta}$. Moreover it follows from Condition $S_{n}$, the continuous embedding $\mathscr{B}_{n} \subseteq \mathscr{B}$, and the closed graph theorem, that there exists a positive function $C_{n}$ such that

But

$$
\left\|S_{t} a\right\|_{n} \leqq C_{n}(t)\|a\|, \quad a \in \mathscr{B}, t>0 .
$$

$$
\left\|S_{t} a\right\|_{n} \leqq C_{n}(s)\left\|S_{t-s} a\right\| \leqq N C_{n}(s) e^{\varrho(t-s)}\|a\|,
$$

for $t \geqq s>0$, and consequently one can assume that $C_{n}$ is uniformly bounded on finite intervals of $\langle 0, \infty\rangle$. Nevertheless the $C_{n}$ can diverge as $t \rightarrow 0$ and the next smoothness condition specifies that the divergence should be similar to those encountered in the group representation case [BGJR].

Condition $\boldsymbol{B}_{n}$. The heat semigroup $S$ satisfies Condition $S_{n}$ and in addition there exists $a C_{n}>0$ such that

$$
\left\|S_{t} a\right\|_{n} \leqq C_{n}\|a\| t^{-n / 2}, \quad a \in \mathscr{B}, t \in\langle 0,1\rangle .
$$

We note that it was established in [BGJR] that if $(\mathscr{B}, g, d U)$ is the representation obtained by differentiating a continuous representation $(\mathscr{B}, G, U)$ of the simply connected Lie group $G$ having $g$ as its Lie algebra, i.e. if $d U(x)$ is the infinitesimal generator of the subgroup $t \rightarrow U\left(e^{t x}\right)$ for each $x \in g$, then Condition $B_{n}$ is satisfied for all $n \geqq 1$. More specifically for each basis $x_{1}, \ldots, x_{d}$ of $g$ there exist $k, \ell>0$ such that

$$
\left\|d U\left(x_{i_{1}}\right) \ldots d U\left(x_{i_{n}}\right) S_{t} a\right\| \leqq k \ell^{n} n !\|a\| t^{-n / 2}
$$

for all $a \in \mathscr{B}, t \in\langle 0,1\rangle$, and $n \geqq 1$. In fact these bounds follow from Conditions $B_{1}$ and $S_{\infty}$ simply by use of the Lie algebraic structure [BGJR, Theorem 2.1]. These remarks motivate much of the following analysis.

First observe that if Condition $B_{2}$ is satisfied then $S$ is automatically holomorphic. This follows because Condition $B_{2}$ implies that $S_{t} \mathscr{B} \subseteq \mathscr{B}_{2} \subseteq D(\Delta)$ and $\left\|\Delta S_{t} a\right\| \leqq d\left\|S_{t} a\right\|_{2} \leqq d C_{2}\|a\| t^{-1}$ for all $a \in \mathscr{B}$, and $t \in\langle 0,1\rangle$. But this is the first basic characterization of holomorphy for $S$. 
Second a variation on the proof of Theorem 2.1 of [BGJR] establishes that Condition $B_{n}$ follows from Condition $B_{1}$ if one knows a priori that Condition $S_{n+1}$ is satisfied.

Proposition 3.1. If Condition $B_{1}$ and $S_{n+1}$ are satisfied then Condition $B_{m}$ is satisfied for $m=1,2, \ldots, n$.

Remarks 3.2. 1. Proposition 3.1 can be considerably strengthened if $n \geqq 3$, see Theorem 6.1 in Sect. 6.

2. If the $V\left(x_{i}\right), i=1,2, \ldots, d$ are $m$-dissipative, i.e. if

$$
\left\|\left(I+\varepsilon V\left(x_{i}\right)\right) a\right\| \geqq m\|a\|
$$

for all $a \in \mathscr{B}_{1}$, small $\varepsilon>0$, and $i=1, \ldots, d$, where $m \in\langle 0,1]$ then Condition $B_{n}$ implies Condition $B_{p}$ for $p=1, \ldots, n$. This converse to Proposition 3.1 follows because $m$ dissipativity implies

$$
\left\|V\left(x_{i}\right) a\right\| \leqq t^{1 / 2} m^{-1}\left\|V\left(x_{i}\right)^{2} a\right\|+\left(1+m^{-1}\right) t^{-1 / 2}\|a\|
$$

for all $a \in \mathscr{B}_{2}, t>0$, and $i=1, \ldots, d$, by the proof of Lemma 2.3 in [Rob2].

We have already noted that Condition $B_{n}$ is always satisfied for the differential $(\mathscr{B}, g, d U)$ of a group representation $(\mathscr{B}, G, U)$ but more is true. The representation $U$ has the property $U \mathscr{B}_{m}(d U)=\mathscr{B}_{m}(d U)$ and the restriction $U_{m}$ of $U$ to $\mathscr{B}_{m}(d U)$ is $\|\cdot\|_{m}$-continuous. Moreover $S \mathscr{B}_{m}(d U) \subseteq \mathscr{B}_{m}(d U)$ and the restriction of $S$ to $\mathscr{B}_{m}(d U)$ is a heat semigroup for the representation $\left(\mathscr{B}_{m}, g, d U_{m}\right)$. Therefore Condition $B_{n}$ is valid for the latter representation and this states that there are $C_{n, m}>0$ such that

$$
\left\|S_{t} a\right\|_{m+n} \leqq C_{n, m}\|a\|_{m} t^{-n / 2}
$$

for all $a \in \mathscr{B}_{m}$ and $t \in\langle 0,1\rangle$. The next proposition establishes that Conditions $B_{1}$ and $S_{n+1}$ imply a weak version of these estimates.

Proposition 3.3. If Conditions $B_{1}$ and $S_{n+1}$ are satisfied then there is a $C_{n}^{\prime}>0$ such that

$$
\left\|S_{t} a\right\|_{n} \leqq C_{n}^{\prime}\|a\|_{n-1} t^{-1 / 2}
$$

for all $a \in \mathscr{B}_{m_{n}}$ and $t \in\langle 0,1\rangle$, where $m_{n}=n$ if $n$ is even and $m_{n}=n+1$ if $n$ is odd.

Proof. Again this follows by a slight variation of the proof of Theorem 2.1 in [BGJR].

Remarks 3.4. 1. Since Condition $S_{n+1}$ implies Condition $S_{n}$ the conclusion of Proposition 3.3 can be iterated, i.e. there are $C_{n, p}^{\prime}>0$ such that

$$
\left\|S_{t} a\right\|_{n} \leqq C_{n, p}^{\prime}\|a\|_{n-p} t^{-p / 2}
$$

for all $a \in \mathscr{B}_{m_{n}}, t \in\langle 0,1\rangle$, and $p=1,2, \ldots, n$. But it does not appear that one can remove the restriction $a \in \mathscr{B}_{m_{n}}$ without assuming $S$ is $\|\cdot\|_{p}$-bounded uniformly for $t$ in a neighbourhood of the origin. Specifically one has the following.

2. If $S$ satisfies Condition $B_{1}$ and $S_{3}$ and in addition $S$ is $\|\cdot\|_{1}$-bounded for $t \in\langle 0,1\rangle$, i.e.

$$
\left\|S_{t} a\right\|_{1} \leqq k\|a\|_{1}
$$


for some $k>0$ and all $t \in\langle 0,1\rangle$, then there is a $C_{2}^{\prime}>0$ such that

$$
\left\|S_{t} a\right\|_{2} \leqq C_{2}^{\prime}\|a\|_{1} t^{-1 / 2}
$$

for all $a \in \mathscr{B}_{1}$ and $t \in\langle 0,1\rangle$. This follows from Proposition 3.3 because $S_{s} a \in \mathscr{B}_{3} \subseteq \mathscr{B}_{2}$ for $0<s<t$ and

$$
\left\|S_{t} a\right\|_{2}=\left\|S_{t-s}\left(S_{s} a\right)\right\|_{2} \leqq C_{2}^{\prime}\left\|S_{s} a\right\|_{1}(t-s)^{-1 / 2} \leqq\left(C_{2}^{\prime} k\right)\|a\|_{1}(t-s)^{-1 / 2} .
$$

Hence taking the limit $s \rightarrow 0$, and redefining $C_{2}^{\prime} k$ as $C_{2}^{\prime}$, one obtains the desired bound.

\section{b) Lipschitz Spaces}

Let $\mathscr{B}_{\alpha, q}^{S}$ denote the Lipschitz spaces defined in Sect. 2 but now $S$ denotes a heat semigroup. Our aim is to give alternative characterizations of the Lipschitz spaces which involve the differential structure of the representation of $g$.

First suppose $S$ satisfies Condition $S_{n}$, then for each $m=1,2, \ldots, n$ we define spaces by

$$
\mathscr{B}_{\alpha, q ; m}=\left\{a \in \mathscr{B} ; t \rightarrow t^{m / 2-\alpha}\left\|S_{t} a\right\|_{m} \in L_{q}(d \mu ;\langle 0,1\rangle)\right\}
$$

with corresponding norms

$$
a \rightarrow\|a\|_{\alpha, q ; m}=\left(\int_{0}^{1} d \mu(t)\left(t^{m / 2-\alpha}\left\|S_{t} a\right\|_{m}\right)^{q}\right)^{1 / q}
$$

if $q<\infty$ and the supremum norm if $q=\infty$. Again it is evident that the $\|\cdot\|_{\alpha, q ; m}$ are seminorms but in fact they are norms, and each $\|\cdot\|_{\alpha, q ; m}$ dominates $\|\cdot\|$. The proof of these statements is completely analogous to the proof of similar statements for the norm (2.8). But now the analogue of the second estimate in (2.11) follows from (3.1) and (3.2), which are implied by Condition $S_{n}$.

It is subsequently useful to note that the norms $\|\cdot\|_{\alpha, q ; m}$ are also equivalent to the norms

$$
a \rightarrow\left(\int_{0}^{\infty} d \mu(t)\left(t^{m / 2-\alpha}\left\|S_{t}^{e} a\right\|_{m} e^{-t}\right)^{q}\right)^{1 / q}
$$

$m=1,2, \ldots, n$, where $S^{\boldsymbol{e}}$ denotes the uniformly bounded semigroup with $S_{t} \boldsymbol{e}$ $=S_{t} \exp \{-\varrho t\}$. Clearly the latter norm dominates $\|\cdot\|_{\alpha, q ; m}$ but one also has

$$
\begin{aligned}
\left(\int_{0}^{\infty} d \mu(t)\left(t^{m / 2-\alpha}\left\|S_{t} e a\right\|_{m} e^{-t}\right)^{q}\right)^{1 / q} \leqq & \|a\|_{\alpha, q ; m} \\
& +N c_{m}(1)\|a\| e^{-e}\left(\int_{1}^{\infty} d \mu(t)\left(t^{m / 2-\alpha} e^{-t}\right)^{q}\right)^{1 / q},
\end{aligned}
$$

where we have used the estimate (3.2). Since the norm $\|\cdot\|$ is dominated by $\|\cdot\|_{\alpha, q ; m}$ by the reasoning of the previous paragraph one now concludes that the norm given by (3.9) is dominated by $\|\cdot\|_{\alpha, q ; m}$. Therefore the norms are equivalent.

Our aim is to identify the $\mathscr{B}_{\alpha, q}^{S}$ and the $\mathscr{B}_{\alpha, q ; m}$ and a third family of spaces which interpolate between $\mathscr{B}$ and the subspaces $\mathscr{B}_{n}$. Theses spaces are defined in terms of 
the interpolation functions

$$
t>0 \rightarrow K_{a}^{(n, m)}(t)=\inf \left\{\left\|a_{0}\right\|+t\left\|a_{m}\right\|_{n} ; a=a_{0}+a_{m}, a_{0} \in \mathscr{B}, a_{m} \in \mathscr{B}_{m}\right\},
$$

where $1 \leqq n \leqq m$ and we set $K_{a}^{(n)}=K_{a}^{(n, n)}$. The corresponding spaces are defined by

$$
\mathscr{B}_{\alpha, q}^{(i)}=\left\{a \in \mathscr{B} ; t \rightarrow t^{-\alpha} K_{a}^{(i)}(t) \in L_{q}(d \mu ;\langle 0,1\rangle)\right\}
$$

and they are Banach spaces with respect to the norms

$$
a \rightarrow\|a\|_{\alpha, q}^{(i)}=\|a\|+\left(\int_{0}^{1} d \mu(t)\left(t^{-\alpha} K_{a}^{(i)}(t)\right)^{q}\right)^{1 / q}
$$

for $q<\infty$ with the obvious modification if $q=\infty$. The spaces $\mathscr{B}_{\alpha, q}^{(n)}=\mathscr{B}_{\alpha, q}^{(n, n)}$ are the standard interpolation spaces between $\mathscr{B}$ and $\mathscr{B}_{n}$ whilst the spaces $\mathscr{B}_{\alpha, q}^{(n, m)}$ interpolate between $\mathscr{B}$ and $\mathscr{B}_{n, m}$, the $\|\cdot\|_{n}$-closure of $\mathscr{B}_{m}$ in $\mathscr{B}_{n}$.

In relating these various spaces we often use two inequalities due to Hardy:

$$
\begin{gathered}
\left(\int_{0}^{1} d \mu(t)\left(t^{-\alpha} \int_{0}^{t} d \mu(s) f(s)^{q}\right)^{1 / q} \leqq \alpha^{-1}\left(\int_{0}^{1} d \mu(t)\left(t^{-\alpha} f(t)\right)^{q}\right)^{1 / q},\right. \\
\left(\int_{0}^{1} d \mu(t)\left(t^{\alpha} \int_{t}^{\infty} d \mu(s) f(s)\right)^{q}\right)^{1 / q} \leqq \alpha^{-1}\left(\int_{0}^{\infty} d \mu(t)\left(t^{\alpha} f(t)\right)^{q}\right)^{1 / q},
\end{gathered}
$$

which are valid for appropriately measurable positive functions $f$ and all $\alpha>0$. In fact these inequalities follow from Minkowski's inequality after a change of variables $s \rightarrow t u$.

Theorem 3.5. If $S$ satisfies Condition $B_{1}$ and Condition $S_{n+1}$ for some $n \geqq 2$ then

for $m=1,2, \ldots, n$.

$$
\mathscr{B}_{\alpha / 2, q}^{S}=\mathscr{B}_{\alpha / 2, q ; m}=\mathscr{B}_{\alpha, q}^{(1, m+1)}
$$

Proof. The proof is in five steps.

Step 1. $\mathscr{B}_{\alpha / 2, q}^{S} \subseteq \mathscr{B}_{\alpha / 2, q ; 1}$.

Let $S^{\varrho}$ denote the semigroup with generator $\bar{\Delta}_{\varrho}=\bar{\Delta}+\varrho I$. Then $\left\|S_{t}^{\varrho}\right\| \leqq N$ for $t>0$ and $S^{\varrho}$ satisfies Condition $B_{1}$. Hence, by use of the Duhamel formula,

$$
\begin{aligned}
\left\|S_{t}^{\varrho} a\right\|_{1} e^{-t} & \leqq \int_{t}^{\infty} d s\left\|S_{s / 2}^{\varrho}\left(I+\Delta_{\varrho}\right) S_{s / 2}^{\varrho} a\right\| e^{-s} \\
& \leqq \sqrt{2 C_{1}} \int_{t}^{\infty} d s s^{-1 / 2}\left\|\left(I+\Delta_{\varrho}\right) S_{s / 2}^{\varrho} a\right\| e^{-s} .
\end{aligned}
$$

Consequently (3.12) implies that

$$
\|a\|_{\alpha / 2, q ; 1} \leqq 2^{(3-\alpha) / 2} C_{1}(1-\alpha)^{-1}\left(\int_{0}^{\infty} d \mu(t)\left(t^{1-\alpha / 2}\left\|\left(I+\Delta_{\varrho}\right) S_{t}^{\varrho} a\right\| e^{-t}\right)^{q}\right)^{1 / q}
$$

But it follows from (2.9), with $T^{\varrho}=S^{\varrho}$ and $H_{e}=\bar{\Delta}_{\varrho}$, that the integrals on the right define a norm equivalent to $\|\cdot\|_{\alpha / 2, q}^{S}$. Therefore $\|\cdot\|_{\alpha / 2, q}^{S}$ dominates $\|\cdot\|_{\alpha / 2, q ; 1}$ and $\mathscr{B}_{\alpha / 2, q}^{S} \subseteq \mathscr{B}_{\alpha / 2, q ; 1}$. 
Step 2. $\mathscr{B}_{\alpha / 2, q ; 1} \subseteq \mathscr{B}_{\alpha / 2, q ; 2} \subseteq \ldots \subseteq \mathscr{B}_{\alpha / 2, q ; n}$.

It follows from Proposition 3.3 that

$$
\left\|S_{t} a\right\|_{m} \leqq C_{m}^{\prime}\left\|S_{t / 2} a\right\|_{m-1}(t / 2)^{-1 / 2}
$$

for all $a \in \mathscr{B}, t \in\langle 0,1\rangle$, and $m=2,3, \ldots, n$. Hence $\|\cdot\|_{\alpha / 2, q ; m-1}$ dominates $\|\cdot\|_{\alpha / 2, q ; m}$ for $m=2,3, \ldots, n$.

Step 3. $\mathscr{B}_{\alpha / 2, q ; n} \subseteq \mathscr{B}_{\alpha / 2, q}^{S}$.

Again let $S^{\varrho}$ denote the modified semigroup with generator $\bar{\Delta}_{\varrho}=\Delta+\varrho I$. We aim to estimate the norm $\|\cdot\|_{\alpha / 2, q}^{S}$ in the equivalent form given by (2.9), with $T^{\varrho}=S^{\varrho}$ and $H_{\varrho}=\bar{\Delta}_{\varrho}$. There are two cases to consider, $n$ odd or $n$ even.

Assume $n$ is odd. Then

$$
\begin{aligned}
\left\|\left(I+\bar{\Delta}_{\varrho}\right)^{(n+1) / 2} S_{t}^{\varrho} a\right\| & =\left\|\left(I+\bar{\Delta}_{\varrho}\right)^{(n-1) / 2} S_{t / 2}^{\varrho}\left(I+\bar{\Delta}_{\varrho}\right) S_{t / 2}^{\varrho} a\right\| \\
& \leqq(1+\varrho+d)^{(n-1) / 2}\left\|S_{t / 2}^{\varrho}\left(I+\bar{\Delta}_{\varrho}\right) S_{t / 2}^{\varrho} a\right\|_{n-1} .
\end{aligned}
$$

Now if $b=\left(I+\bar{\Delta}_{\varrho}\right) S_{t / 2}^{\varrho} a$, then $b=S_{t / 4}^{\varrho}\left(I+\bar{\Delta}_{\varrho}\right) S_{t / 4}^{\varrho} a \in \mathscr{B}_{n+1} \subseteq \mathscr{B}_{n}$, and it follows from Proposition 3.3 that

$$
\begin{aligned}
\left\|S_{t / 2}^{\varrho}\left(I+\bar{\Delta}_{\varrho}\right) S_{t / 2}^{\varrho} a\right\|_{n-1} & \leqq C_{n-1}^{\prime}\left\|\left(I+\bar{\Delta}_{\varrho}\right) S_{t / 2}^{\varrho} a\right\|_{n-2}(t / 2)^{-1 / 2} \\
& \leqq \sqrt{2}(1+\varrho+d) C_{n-1}^{\prime}\left\|S_{t / 2}^{\varrho} a\right\|_{n} t^{-1 / 2}
\end{aligned}
$$

for all $t \in\langle 0,1\rangle$. Therefore combining (3.13) and (3.14) gives

$$
\left\|\left(I+\bar{\Delta}_{\varrho}\right)^{(n+1) / 2} S_{t}^{\varrho} a\right\| \leqq \sqrt{2}(1+\varrho+d)^{(n+1) / 2} C_{n-1}^{\prime}\left\|S_{t / 2}^{\varrho} a\right\|_{n} t^{-1 / 2}
$$

for $t \in\langle 0,1\rangle$. But Condition $B_{2}$ is satisfied by Proposition 3.1 and hence

$$
\begin{aligned}
\left\|\left(I+\bar{\Delta}_{\varrho}\right)^{(n+1) / 2} S_{t}^{\varrho} a\right\| & \leqq\left\|\left(I+\bar{\Delta}_{\varrho}\right) S_{2 /(n+1)}^{\varrho}\right\|^{(n+1) / 2} \cdot\left\|S_{t-1}^{\varrho} a\right\| \\
& \leqq k_{n}\|a\|
\end{aligned}
$$

for all $t>1$. One immediately concludes from these estimates that there are $C_{\alpha, q}$, $d_{\alpha, q}>0$ such that

$$
\left(\int_{0}^{\infty} d \mu(t)\left(t^{(n+1-\alpha) / 2}\left\|\left(I+\bar{\Delta}_{\varrho}\right)^{(n+1) / 2} S_{t}^{\varrho} a\right\| e^{-t}\right)^{q}\right)^{1 / q} \leqq C_{\alpha, q}\|a\|+d_{\alpha, q}\|a\|_{\alpha / 2, q ; n} .
$$

But the norm $\|\cdot\|$ is dominated by $\|\cdot\|_{\alpha / 2, q ; n}$ and therefore $\|\cdot\|_{\alpha / 2, q}^{S}$ is dominated by $\|\cdot\|_{\alpha / 2, q ; n}$.

Next assume $n$ is even. Then

$$
\left\|\left(I+\bar{\Delta}_{\varrho}\right)^{n / 2} S_{t}^{\varrho} a\right\| \leqq(1+\varrho+d)^{n}\left\|S_{t}^{\varrho} a\right\|_{n}
$$

and the estimate proceeds as above with the norm corresponding to (2.9) with $m=n / 2$.

Step 4. $\mathscr{B}_{\alpha / 2, q}^{S} \subseteq \mathscr{B}_{\alpha, q}^{(1, n+1)} \subseteq \ldots \subseteq \mathscr{B}_{\alpha, q}^{(1,2)}$.

Since $S_{t} \mathscr{B} \subseteq \mathscr{B}_{n+1}$ for $t>0$,

$$
K_{a}^{(1,2)}(t) \leqq \ldots \leqq K_{a}^{(1, n+1)}(t) \leqq\left\|\left(I-S_{t^{2}}\right) a\right\|+t\left\|S_{t^{2}} a\right\|_{1} .
$$


Hence by Minkowski's inequality

$$
\|a\|_{\alpha, q}^{(1,2)} \leqq \ldots \leqq\|a\|_{\alpha, q}^{(1, n+1)} \leqq(1 / 2)\|a\|_{\alpha / 2, q}^{S}+\|a\|_{\alpha, q ; 1}
$$

Since $\|\cdot\|_{\alpha, q ; 1}$ is equivalent to $\|\cdot\|_{\alpha / 2, q}^{S}$ by the previous steps the desired result follows immediately.

Step 5. $\mathscr{B}_{\alpha, q}^{(1,2)} \subseteq \mathscr{B}_{\alpha / 2, q}^{S}$.

First, if $a_{0} \in \mathscr{B}$ then

$$
\left\|\left(I-S_{t^{2}}\right) a_{0}\right\| \leqq\left(1+N e^{\varrho}\right)\left\|a_{0}\right\|
$$

for all $t \in\langle 0,1\rangle$. Second, if $a_{2} \in \mathscr{B}_{2}$ then

$$
\begin{aligned}
\left\|\left(I-S_{t^{2}}\right) a_{2}\right\| & \leqq d \int_{0}^{t^{2}} d s\left\|S_{s} a_{2}\right\|_{2} \\
& \leqq 2 d C_{1}^{\prime}\left\|a_{2}\right\|_{1} t
\end{aligned}
$$

by Duhamel's formula and Proposition 3.3. Consequently if $a=a_{0}+a_{2}$,

$$
\left\|\left(I-S_{t^{2}}\right) a\right\| \leqq C K_{a}^{(1,2)}(t),
$$

where $C=\max \left(\left(1+N e^{\varrho}\right), 2 d C_{1}^{\prime}\right)$. Therefore $\mathscr{B}_{\alpha, q}^{(1,2)} \subseteq \mathscr{B}_{\alpha / 2, q}^{S}$.

The above proof is only valid for $q<\infty$ but the case $q=\infty$ is similar but simpler. We omit the details.

Corollary 3.6. If $S$ satisfies Conditions $B_{1}$ and $S_{3}$ and if, in addition $S$ is $\|\cdot\|_{1}$-bounded uniformly for $t \in\langle 0,1\rangle$, i.e. $\left\|S_{t} a\right\|_{1} \leqq k\|a\|_{1}$ for all $a \in \mathscr{B}_{1}$ and $t \in\langle 0,1\rangle$, then

$$
\mathscr{B}_{\alpha / 2, q}^{S}=\mathscr{B}_{\alpha, q}^{(1)} \text {. }
$$

Proof. It follows from Step 4 of the above proof that $\mathscr{B}_{\alpha / 2, q}^{S} \subseteq \mathscr{B}_{\alpha, q}^{(1)}$. But then by Remark 3.4.2 one has a bound

$$
\left\|S_{t} a\right\|_{2} \leqq C_{2}^{\prime}\|a\|_{1} t^{-1 / 2}
$$

for all $a \in \mathscr{B}_{1}$ and $t \in\langle 0,1\rangle$. Hence $\mathscr{B}_{\alpha, q}^{(1)} \subseteq \mathscr{B}_{\alpha / 2, q}^{S}$ by the proof in Step 5 .

Finally we identify the spaces $\mathscr{B}_{\alpha, q}^{S}$ with a family of spaces which involve the action of the Poisson semigroup $P$ associated with the heat semigroup $S$ and the differential structure of the representation. The semigroup $P$ is now defined as the semigroup with generator $(\hat{A}+\varrho I)^{1 / 2}$. Hence it is given in terms of $S$ by the analogue of (2.12)

$$
P_{t}=(4 \pi)^{-1 / 2} t \int_{0}^{\infty} d s v_{t}(s) S_{s} e^{-\varrho s},
$$

where $v_{t}(s)=s^{-3 / 2} \exp \left\{-t^{2} / 4 s\right\}$. It inherits smoothness properties from $S$.

Lemma 3.7. If $S$ satisfies Condition $B_{n}$ then $P_{t} \mathscr{B} \subseteq \mathscr{B}_{n}$ for all $t>0$ and there is a $C_{n}^{\prime \prime}$ such that

for all $a \in \mathscr{B}$ and $t \in\langle 0,1\rangle$.

$$
\left\|P_{t} a\right\|_{n} \leqq C_{n}^{\prime \prime}\|a\| t^{-n}
$$

Proof. If $t \in\langle 0,1\rangle$ then

$$
\left\|S_{t} a\right\|_{n} e^{-e t} \leqq C_{n}\|a\| t^{-n / 2}
$$


by Condition $B_{n}$, but if $t \geqq 1$

$$
\left\|S_{t} a\right\|_{n} e^{-\varrho} \leqq C_{n}\left\|S_{t-1} a\right\| e^{-\varrho t} \leqq C_{n} N\|a\| e^{-t} .
$$

But now it follows from (3.15) by a standard closure argument that $P_{t} \mathscr{B} \subseteq \mathscr{B}_{n}$ for $t>0$ and

for $t \in\langle 0,1\rangle$.

$$
\begin{aligned}
\left\|P_{t} a\right\|_{n} & \leqq \int_{0}^{\infty} d s v_{t}(s) N C_{n}\left(1+s^{-n / 2}\right)\|a\| \\
& \leqq C_{n}^{\prime \prime}\|a\| t^{-n}
\end{aligned}
$$

Lemma 3.8. If $S$ satisfies Condition $B_{1}$ and Condition $S_{n+1}$ with $n \geqq 2$ then there is a $C_{n}^{\prime \prime \prime}>0$ such that

$$
\left\|P_{t} a\right\|_{n} \leqq C_{n}^{\prime \prime \prime}\|a\|_{n-1} t^{-1}
$$

for all $a \in \mathscr{B}_{m_{n}}$ and $t \in\langle 0,1\rangle$, where $m_{n}=n$ is even and $m_{n}=n+1$ if $n$ is odd.

This result follows from Proposition 3.3 and (3.15) by the same estimates used to prove Lemma 3.6.

Now we are prepared to introduce the new family of spaces associated with $P$ by

$$
\mathscr{B}_{\alpha, q ; m}^{\prime}=\left\{a \in \mathscr{B} ; t \rightarrow t^{m-\alpha}\left\|P_{t} a\right\|_{m} \in L_{q}(d \mu ;\langle 0,1\rangle)\right\}
$$

with norms $\|\cdot\|_{\alpha, q ; m}^{\prime}$ given by

$$
\|a\|_{\alpha, q ; m}^{\prime}=\left(\int_{0}^{1} d \mu(t)\left(t^{m-\alpha}\left\|P_{t} a\right\|_{m}\right)^{q}\right)^{1 / q}
$$

if $q<\infty$ and with the supremum norm if $q=\infty$. It follows once again by the closed graph theorem that the $\|\cdot\|_{\alpha, q ; m}^{\prime}$ dominate $\|\cdot\|$.

Theorem 3.9. If $S$ satisfies Condition $B_{1}$ and Condition $S_{n+1}$ for some $n \geqq 2$ then

for $m=1,2, \ldots, m$.

$$
\mathscr{B}_{\alpha / 2, q}^{S}=\mathscr{B}_{\alpha, q ; m}^{\prime}
$$

Proof. Since $\mathscr{B}_{\alpha \mid 2, q}^{S}=\mathscr{B}_{\alpha, q}^{P}$ it suffices to prove that $\mathscr{B}_{\alpha, q}^{P}=\mathscr{B}_{\alpha, q ; m}^{\prime}, m=1,2, \ldots, n$. The proof is in three steps which are essentially a repetition of Steps 1,2, and 3, in the proof of Theorem 3.5 but now the uniformly bounded semigroup $S^{e}$ with generator $\bar{\Delta}_{e}$ is replaced by the Poisson semigroup $P$ with generator $\bar{\Delta}_{e}^{1 / 2}$. The equivalent norm (2.9) used in the previous proof is then replaced by its analogue in (2.15). Moreover Condition $B_{n}$ and the estimates of Proposition 3.3 are replaced by the statements of Lemmas 3.7 and 3.8. We omit further details.

\section{A Priori Estimates}

In this section we examine estimates for the operators $V(x)$ and their products $V(x) V(y)$ on the Lipschitz spaces $\mathscr{B}_{\alpha / 2, q}^{S}$. Our principal aim is to show that the $V(x)$ are relatively bounded by $\nabla=(\hat{\Delta}+\varrho I)^{1 / 2}$, the generator of the Poisson semigroup and that the products $V(x) V(y)$ are relatively bounded by $\hat{\Delta}$. These estimates are 
similar to those derived earlier in the group representation case [RobI] but now they are established from minimal smoothness hypotheses on the heat semigroup. They reflect the increased regularity of the Lipschitz spaces and the corresponding representation of $g$.

Theorem 4.1. Assume $S$ satisfies Conditions $B_{1}$ and $S_{3}$, then there is a $k_{\alpha, q}>0$ such that

$$
\sup _{1 \leqq i \leqq d}\left\|V\left(x_{i}\right) a\right\|_{\alpha / 2, q}^{S} \leqq k_{\alpha, q}\|(I+\nabla) a\|_{\alpha / 2, q}^{S}
$$

for all $a \in \mathscr{B}_{3}$, where $x_{1}, \ldots, x_{d}$ is a basis of $g$.

If in addition $S$ satisfies Condition $S_{4}$ then there is an $l_{\alpha, q}>0$ such that

$$
\sup _{1 \leqq i, j \leqq d}\left\|V\left(x_{i}\right) V\left(x_{j}\right) a\right\|_{\alpha / 2, q}^{S} \leqq l_{\alpha, q}\left\|\left(I+\bar{\Delta}_{\ell}\right) a\right\|_{\alpha / 2, q}^{S} .
$$

for all $a \in \mathscr{B}_{4}$, where $\bar{\Delta}_{\varrho}=\bar{\Delta}+\varrho I$.

Proof. The proof is accomplished by estimates similar to those used to establish Theorems 6.2 and 6.3 in [RobI]. For brevity we omit the details.

Next we remark that one can also establish converses to (4.1) and (4.2). The converse to (4.2) is the easiest to discuss and can be obtained in a strong form. For example, if

then

$$
\mathscr{B}_{2 ; \alpha, q}=\left\{a \in \mathscr{B}_{2} ; V(x) V(y) a \in \mathscr{B}_{\alpha, q}^{S}, x, y \in g\right\}
$$

$$
\|\Delta a\|_{\alpha / 2, q}^{S} \leqq \sum_{i=1}^{d}\left\|V\left(x_{i}\right)^{2} a\right\|_{\alpha / 2, q}^{S} \leqq d \sup _{1 \leqq i, j \leqq d}\left\|V\left(x_{i}\right) V\left(x_{j}\right) a\right\|_{\alpha / 2, q}^{S}
$$

for all $a \in \mathscr{B}_{2 ; \alpha / 2, q}$, and in particular for all $a \in \mathscr{B}_{4}$. In analogy one might expect a converse to (4.1) for those $a \in \mathscr{B}_{1} \bigcap \mathscr{B}_{\alpha / 2, q}^{S}$ such that $V(x) a \in \mathscr{B}_{\alpha / 2, q}^{S}$ for all $x \in g$. But the difficulty is to first establish that these $a$ are in $D(\nabla)$. This apparently requires stronger continuity hypotheses on $S$ (see Theorem 4.5 below). Nevertheless one does have the following strict converse of (4.1).

Proposition 4.2. Assume $S$ satisfies Conditions $B_{1}$ and $S_{3}$. Then there is a $k_{\alpha, q}^{\prime}>0$ such that

$$
\|\nabla a\|_{\alpha / 2, q}^{S} \leqq k_{\alpha, q}^{\prime}\left(\|a\|_{\alpha / 2, q}^{S}+\sup _{1 \leqq i \leqq d}\left\|V\left(x_{i}\right) a\right\|_{\alpha / 2, q}^{S}\right)
$$

for all $a \in \mathscr{B}_{3}$, where $x_{1}, \ldots, x_{d}$ is a basis of $g$.

Proof. In order to establish (4.4) it is first useful to note that one can effectively replace $\nabla$ by $\left(I+\bar{\Delta}_{\varrho}\right)^{1 / 2}$, where $\bar{\Delta}_{\varrho}=\bar{\Delta}+\varrho I$. This is a consequence of standard semigroup estimates.

Lemma 4.3. Let $T$ be a strongly continuous semigroup with generator $H$ satisfying the uniform bound $\left\|T_{t}\right\| \leqq N$. It follows that $D\left(H^{1 / 2}\right)=D\left((I+H)^{1 / 2}\right)$ and

$$
\left\|(I+H)^{1 / 2} a-H^{1 / 2} a\right\| \leqq N\|a\|
$$

for all $a \in D\left(H^{1 / 2}\right)$. Consequently

$$
\left\|H^{1 / 2} a\right\| \leqq\left(1+N^{2}\right)\left\|(I+H)^{1 / 2} a\right\|
$$

for all $a \in D\left(H^{1 / 2}\right)$. 
Proof. The proof follows by simple estimation once one remarks that

$$
H^{1 / 2} a=\lim _{\varepsilon \rightarrow 0} \Gamma(-1 / 2)^{-1} \int_{\varepsilon}^{\infty} d \mu(t) t^{-1 / 2}\left(I-T_{t}\right) a
$$

where the limit exists if, and only if, $a \in D\left(H^{1 / 2}\right)$ (see, for example, [Yos] but note that we use a different convention for the sign of the generator). Similarly

$$
(I+H)^{1 / 2} a=\lim _{\varepsilon \rightarrow 0} \Gamma(-1 / 2)^{-1} \int_{\varepsilon}^{\infty} d \mu(t) t^{-1 / 2}\left(I-T_{t} e^{-t}\right) a,
$$

where once again the limit exists if, and only if, $a \in D\left((I+H)^{1 / 2}\right)$.

Now let us return to the proof of (4.4).

It follows from Lemma 4.3 that

$$
\begin{aligned}
\left\|\left(I-S_{t}\right) \nabla a\right\| & =\left\|\nabla\left(I-S_{t}\right) a\right\| \\
& \leqq\left(1+N^{2}\right)\left\|\left(I-S_{t}\right)\left(I+\bar{\Delta}_{\varrho}\right)^{1 / 2} a\right\|
\end{aligned}
$$

for all $a \in D(\nabla)$. Consequently

$$
\|\nabla a\|_{\alpha, q}^{S} \leqq\left(1+N^{2}\right)\left\|\left(I+\bar{\Delta}_{\varrho}\right)^{1 / 2} a\right\|_{\alpha, q}^{S}
$$

for all $a \in D(\nabla)$ such that $\nabla a \in \mathscr{B}_{\alpha, q}^{S}$. In particular (4.7) is valid for all $a \in \mathscr{B}_{3}$.

Next remark that $\|\cdot\|_{\alpha, q}^{\boldsymbol{P}}$, and hence $\|\cdot\|_{\alpha / 2, q}^{S}$, is equivalent to the norm

$$
a \rightarrow\|a\|_{\alpha, q}^{\Delta}=\left(\int_{0}^{1} d \mu(t)\left(t^{1-\alpha}\left\|\left(I+\bar{\Delta}_{\varrho}\right)^{1 / 2} Q_{t} a\right\|\right)^{q}\right)^{1 / q}
$$

where $Q$ is the Poisson semigroup with generator $\left(I+\bar{\Delta}_{\varrho}\right)^{1 / 2}$ associated with the semigroup $t \rightarrow S_{t}^{1+\varrho}=S_{t} \exp \{-(1+\varrho) t\}$. This equivalence of norms follows from the discussion at the end of Sect. 2. Therefore

$$
\begin{aligned}
\left\|\left(I+\bar{\Delta}_{\varrho}\right)^{1 / 2} a\right\|_{\alpha, q}^{\Delta} & =\left(\int_{0}^{1} d \mu(t)\left(t^{1-\alpha}\left\|\left(I+\bar{\Delta}_{\varrho}\right) Q_{t} a\right\|\right)^{q}\right)^{1 / q} \\
& \leqq C_{\alpha, q}\|a\|+\left(\int_{0}^{1} d \mu(t)\left(t^{1-\alpha}\left\|\bar{\Delta} Q_{t} a\right\|\right)^{q}\right)^{1 / q}
\end{aligned}
$$

for a suitable $C_{\alpha, q}>0$. But

$$
\left\|\bar{\Delta} Q_{t} a\right\| \leqq \sum_{i=1}^{d}\left\{\left\|Q_{t} V\left(x_{i}\right) a\right\|_{1}+\left\|\left(\operatorname{ad} Q_{t}\right)\left(V\left(x_{i}\right)\right) a\right\|_{1}\right\} .
$$

Moreover it follows from Theorem 3.8, applied to the pair $S^{1+e}$ and $Q$, that

$$
\left(\int_{0}^{1} d \mu(t)\left(t^{1-\alpha} \sum_{i=1}^{d}\left\|Q_{t} V\left(x_{i}\right) a\right\|_{1}\right)^{q}\right)^{1 / q} \leqq d_{\alpha, q} \sum_{i=1}^{d}\left\|V\left(x_{i}\right) a\right\|_{\alpha / 2, q}^{S}
$$

for a suitable $d_{\alpha, q}>0$. Next one has

$$
\left\|\left(\operatorname{ad} Q_{t}\right)\left(V\left(x_{i}\right)\right) a\right\|_{1} \leqq \int_{0}^{\infty} d s v_{t}(s) e^{-(1+\varrho) s}\left\|\left(\operatorname{ad} S_{s}\right)\left(V\left(x_{i}\right)\right) a\right\|_{1} .
$$


But since $a \in \mathscr{B}_{3}$ and $S_{t} a \in \mathscr{B}_{3}$ for $t>0$

$$
\left(\operatorname{ad} S_{s}\right)\left(V\left(x_{i}\right)\right) a=\int_{0}^{s} d u S_{s-u}(\operatorname{ad} \bar{\Delta})\left(V\left(x_{i}\right)\right) S_{u} a
$$

Now $(\operatorname{ad} \Delta)\left(V\left(x_{i}\right)\right)$ is of second order in the $V\left(x_{j}\right)$ and hence

$$
\begin{aligned}
\left\|\left(\operatorname{ad} S_{s}\right)\left(V\left(x_{i}\right)\right) a\right\|_{1} & \leqq \int_{0}^{s} d u\left\|S_{s-u}(\operatorname{ad} \bar{\Delta})\left(V\left(x_{i}\right)\right) S_{u} a\right\|_{1} \\
& \leqq C \int_{0}^{s} d u(s-u)^{-1 / 2}\left\|S_{u} a\right\|_{2}
\end{aligned}
$$

for a suitable $c>0$. Therefore combining (4.11) and (4.12) one obtains the estimate

$$
\begin{aligned}
\left\|\left(\operatorname{ad} Q_{t}\right)\left(V\left(x_{i}\right)\right)\right\|_{1} & \leqq C \int_{0}^{\infty} d s v_{t}(s) s^{1 / 2} \int_{0}^{1} d u(1-u)^{-1 / 2}\left\|S_{s u}^{1+\varrho} a\right\|_{2} \\
& =C \pi^{-1 / 2} t \int_{0}^{\infty} d v v^{-1} e^{-v / 4} \int_{0}^{1} d u(1-u)^{-1 / 2}\left\|S_{t^{2} u / v}^{1+e} a\right\|_{2}
\end{aligned}
$$

Therefore, by the Minkowski inequality,

$$
\begin{aligned}
& \left(\int_{0}^{1} d \mu(t)\left(t^{1-\alpha}\left\|\left(\operatorname{ad} Q_{t}\right)\left(V\left(x_{i}\right)\right) a\right\|_{1}\right)^{q}\right)^{1 / q} \\
& \leqq C \pi^{-1 / 2} \int_{0}^{\infty} d v v^{-1} e^{-v / 4} \int_{0}^{1} d u(1-u)^{-1 / 2}\left(\int_{0}^{1} d \mu(t)\left(t^{2-\alpha}\left\|S_{t^{2} u / v}^{1+e} a\right\|_{2}\right)^{q}\right)^{1 / q} \\
& \leqq C(4 \pi)^{-1 / 2} \int_{0}^{\infty} d v v^{-\alpha / 2} e^{-v / 4} \int_{0}^{1} d u u^{-1+\alpha / 2}(1-u)^{-1 / 2} \\
& \quad \cdot\left(\int_{0}^{\infty} d \mu(t)\left(t^{1-\alpha / 2}\left\|S_{t}^{\varrho} a\right\|_{2} e^{-t}\right)^{q}\right)^{1 / q} .
\end{aligned}
$$

But the first two integrals converge and the third integral defines a norm equivalent to $\|\cdot\|_{\alpha / 2, q ; 2}$ and hence $\|\cdot\|_{\alpha / 2, q}^{S}$, by the discussion at the beginning of Sect. $3 \mathrm{~b}$ and Theorem 3.4. Finally (4.4) follows from (4.8)-(4.13) and the equivalence of the various norms involved.

Next we aim to strengthen the conclusion of Proposition 4.2 by exploitation of a stronger continuity hypothesis for $S$. But as a preliminary it is useful to note that the estimates (4.1) and (4.4) can be rephrased as an equivalence of norms.

Lemma 4.4. Assume $S$ satisfies Condition $B_{1}$ and $S_{3}$. Then the following norms are equivalent on $\mathscr{B}_{3}$ :

$$
\begin{aligned}
& a \in \mathscr{B}_{3} \rightarrow\|a\|_{\alpha / 2, q}^{S}+\sup _{1 \leqq i \leqq d}\left\|V\left(x_{i}\right) a\right\|_{\alpha / 2, q}^{S}, \\
& a \in \mathscr{B}_{3} \rightarrow\|a\|_{1}+\left(\int_{0}^{1} d \mu(t)\left(t^{-\alpha / 2}\left\|\left(I-S_{t}\right) a\right\|_{1}\right)^{q}\right)^{1 / q} .
\end{aligned}
$$


Proof. First note $\mathscr{B}_{\alpha / 2, q}^{S}=\mathscr{B}_{\alpha, q}^{(1,3)}$ by Theorem 3.5. Hence there is a $C_{\alpha, q}>0$ such that

$$
\|a\|_{\alpha / 2, q}^{S} \leqq C_{\alpha, q}\|a\|_{\alpha, q}^{(1,3)}
$$

But for $a \in \mathscr{B}_{3}$ one has

$$
\|a\|_{\alpha, q}^{(1,3)} \leqq\left(\int_{0}^{1} d \mu(t) t^{q(1-\alpha)}\right)^{1 / q}\|a\|_{1} .
$$

Thus for all $a \in \mathscr{B}_{3}$ one has estimates

$$
\|a\|_{\alpha / 2, q}^{S} \leqq C_{\alpha, q}^{\prime}\|a\|_{1} \leqq C_{\alpha, q}^{\prime}\left(\|a\|_{\alpha / 2, q}^{S}+\sup _{1 \leqq i \leqq d}\left\|V\left(x_{i}\right) a\right\|_{\alpha / 2, q}^{S}\right)
$$

Second since $S$ satisfies Condition $S_{3}$ one has

$$
S_{t} V(x) a=V(x) S_{t} a+\int_{0}^{1} d s S_{t-s}(\operatorname{ad} \bar{\Delta})(V(x)) S_{s} a
$$

for all $a \in \mathscr{B}_{3}$ and $x \in g$. Hence there is a $C>0$ such that

$$
\left\|\left(I-S_{t}\right) V(x) a\right\|-\left\|V(x)\left(I-S_{t}\right) a\right\| \mid \leqq C \int_{0}^{t} d s\left\|S_{s} a\right\|_{2}
$$

for all $t \in\langle 0,1\rangle$. Consequently

$$
\begin{aligned}
\left|\left(\int_{0}^{1} d \mu(t)\left(t^{-\alpha / 2}\left\|\left(I-S_{t}\right) V(x) a\right\|\right)^{q}\right)^{1 / q}-\left(\int_{0}^{1} d \mu(t)\left(t^{-\alpha / 2}\left\|V(x)\left(I-S_{t}\right) a\right\|\right)^{q}\right)^{1 / q}\right| \\
\leqq C(1-\alpha)^{-1}\left(\int_{0}^{1} d \mu(t)\left(t^{1-\alpha / 2}\left\|S_{t} a\right\|_{2}\right)^{q}\right)^{1 / q} \\
\leqq C^{\prime}\|a\|_{\alpha / 2, q}^{S}
\end{aligned}
$$

by another application of Theorem 3.5.

Finally the statement of the lemma follows straightforwardly from combination of (4.14) and (4.15).

The interest of Lemma 4.4 rests on the observations that the second norm of the lemma can be identified as a Lipschitz norm with respect to the Banach space $\mathscr{B}_{1}$. Then it is natural to examine the space of elements for which this norm is finite. Theorem 4.1 and Lemma 4.4 indicates that this space contains $D(\nabla)$ and the next theorem elaborates this connection. In this theorem we identify $D(\nabla)_{\alpha, q}$ as the subspace of $\mathscr{B}_{\alpha, q}^{S}$ formed by those $a \in D(\nabla)$ such that $\nabla a \in \mathscr{B}_{\alpha, q}^{S}$ equipped with the norm $a \rightarrow\|(I+\nabla) a\|_{\alpha, q}^{S}$. Moreover we define

$$
\mathscr{B}_{1 ; \alpha, q}^{S}=\left\{a \in \mathscr{B}_{1} \bigcap \mathscr{B}_{\alpha, q}^{S} ; \int_{0}^{1} d \mu(t)\left(t^{-\alpha}\left\|\left(I-S_{t}\right) a\right\|_{1}\right)^{q}<\infty\right\}
$$

equipped with the norm

$$
\|a\|_{1 ; \alpha, q}^{S}=\|a\|_{1}+\left(\int_{0}^{1} d \mu(t)\left(t^{-\alpha}\left\|\left(I-S_{t}\right) a\right\|_{1}\right)^{q}\right)^{1 / q} .
$$


Theorem 4.5. Assume $S$ satisfies Condition $B_{1}$ and $S_{3}$ and that $\alpha \in\langle 0,1 / 2\rangle$, $q \in\langle 1, \infty\rangle$. It follows that

$$
D(\nabla)_{\alpha, q} \subseteq \mathscr{B}_{1 ; \alpha, q}^{S} .
$$

If, in addition, $S$ restricted to $\mathscr{B}_{1}$ is $\|\cdot\|_{1}$-continuous, then

$$
D(\nabla)_{\alpha, q}=\mathscr{B}_{1 ; \alpha, q}^{S} .
$$

Proof. First, from Theorem 4.1, Proposition 4.2, Lemma 4.4, and the dissipativity estimates $\|(I+\nabla) a\|_{\alpha, q}^{S} \geqq N^{-1}\|a\|_{\alpha, q}^{S}$ one obtains bounds

$$
C_{\alpha, q}\|a\|_{1 ; \alpha, q}^{S} \leqq\|(I+\nabla) a\|_{\alpha, q}^{S} \leqq C_{\alpha, q}^{\prime}\|a\|_{1 ; \alpha, q}^{S}
$$

for all $a \in \mathscr{B}_{3}$, and suitable $C_{\alpha, q}, C_{\alpha, q}^{\prime}>0$. Now the two statements of the theorem are obtained by extending these bounds by continuity.

The semigroup $S$ leaves $\mathscr{B}_{\alpha, q}^{S}$ invariant and since $q<\infty$ it follows the Lebesgue dominated convergence theorem that the restriction of $S$ to $\mathscr{B}_{\alpha, q}^{S}$ is $\|\cdot\| \|_{\alpha, q^{-}}^{S}$ continuous. Let $\nabla_{\alpha, q}$ denote the generator of the restricted semigroup. Next as $S_{t} \mathscr{B} \subseteq \mathscr{B}_{3} \subseteq D(\Delta) \subseteq \mathscr{B}_{\alpha, q}$ for all $t>0$ it follows that

$$
\mathscr{D}=\bigcup_{t>0} S_{t} \mathscr{B}_{\alpha, q}^{S}
$$

is a $\|\cdot\| \|_{\alpha, q}^{S}$-dense $S$-invariant subspace of $\Delta_{\alpha, q}$ and hence a core of $\Delta_{\alpha, q}$. But $\mathscr{D} \subseteq \mathscr{B}_{3} \subseteq D\left(\Delta_{\alpha, q}\right)$ and consequently $\mathscr{B}_{3}$ is a core of $\Delta_{\alpha, q}$. Now by general semigroup theory each core of $\Delta_{\alpha, q}$ is a core of $\Delta_{\alpha, q}=\left(\Delta_{\alpha, q}+\varrho I\right)^{1 / 2}$ and $\nabla_{\alpha, q}$ is the restriction of $\nabla$ to $D(\nabla)_{\alpha, q}$. Hence $\mathscr{B}_{3}$ is dense in $D(\nabla)_{\alpha, q}$ with respect to the norm $a \rightarrow\|(I+\nabla) a\|_{\alpha, q}^{S}$. Therefore the estimate on the left of (4.18) extends to all $a \in D(\nabla)_{\alpha, q}$ by continuity and one has $D(\nabla)_{\alpha, q} \subseteq \mathscr{B}_{1 ; \alpha, q}^{S}$.

Conversely, if $S$ is $\|\cdot\|_{1}$-continuous there are $N_{1} \geqq 1$ and $\varrho_{1} \geqq 0$ such that $\left\|S_{t}\right\|_{1} \leqq N_{1} \exp \left\{\varrho_{1} t\right\}$ for all $t>0$. Therefore

$$
\left\|\left(I-S_{t}\right) S_{s} a\right\|_{1} \leqq N_{1} e^{\varrho_{1} s}\left\|\left(I-S_{t}\right) a\right\|_{1}
$$

and in particular $\mathscr{B}_{1 ; \alpha, q}^{S}$ is $S$-invariant. But it also follows from $\|\cdot\|_{1}$-continuity that

$$
\lim _{s \rightarrow 0}\left\|\left(I-S_{t}\right)\left(I-S_{s}\right) a\right\|_{1}=0 \text {. }
$$

Hence, by the Lebesgue dominated convergence theorem, $S$ restricted to $\mathscr{B}_{1 ; \alpha, q}^{S}$ is $\|\cdot\| \|_{1 ; \alpha, q}^{S}$-continuous. Now since $S_{t} \mathscr{B}_{1 ; \alpha, q}^{S} \subseteq \mathscr{B}_{3} \subseteq \mathscr{B}_{1 ; \alpha, q}^{S}$ it follows that $\mathscr{B}_{3}$ is $\|\cdot\|_{1 ; \alpha, q^{-}}$ dense in $\mathscr{B}_{1 ; \alpha, q}^{S}$. Therefore the estimate on the right of (4.18) extends to all $a \in \mathscr{B}_{1 ; \alpha, q}^{S}$ by continuity and one has $\mathscr{B}_{1 ; \alpha, q}^{S} \subseteq D(\nabla)_{\alpha, q}$.

Finally we note that the spaces $\mathscr{B}_{1 ; \alpha, q}^{S}$ correspond to the Lipschitz spaces of $S$ restricted to $\mathscr{B}_{1}$ and they can be identified as interpolation spaces between $\mathscr{B}_{1}$ and $\mathscr{B}_{2}$. In fact if $S_{t} \mathscr{B} \subseteq \mathscr{B}_{3}$ for $t>0$ then the spaces interpolate between $\mathscr{B}_{1}$ and the $\|\cdot\|_{2}$-closure of $\mathscr{B}_{3}$ in $\mathscr{B}_{2}$.

\section{Lipschitz Representations}

In this section we demonstrate, under mild smoothness hypotheses on $S$, that the representation of $g$ on $\mathscr{B}$ defines a representation on each Lipschitz space, by restriction. In addition we analyze the regularity properties of these representations. 
First define $V_{\alpha, q}(x), x \in g$, on $\mathscr{B}_{\alpha, q}^{S}$ by

and

$$
D\left(V_{\alpha, q}(x)\right)=\left\{a \in D(V(x)) \cap \mathscr{B}_{\alpha, q}^{S} ; V(x) a \in \mathscr{B}_{\alpha, q}^{S}\right\}
$$

$$
V_{\alpha, q}(x) a=V(x) a
$$

for all $a \in D\left(V_{\alpha, q}(x)\right)$. Since the $V(x)$ are norm-closed and $\mathscr{B}_{\alpha, q}^{S}$ is continuously embedded in $\mathscr{B}$ it follows that the $V_{\alpha, q}(x)$ are $\|\cdot\|_{\alpha, q}^{S}$-closed.

Second introduce the sequence of subspaces

$$
\mathscr{B}_{n ; \alpha, q}=\bigcap_{1 \leqq i_{1}, \ldots, i_{n} \leqq d} D\left(V_{\alpha, q}\left(x_{i_{1}}\right) \ldots V_{\alpha, q}\left(x_{i_{n}}\right)\right),
$$

where $x_{1}, \ldots, x_{d}$ is a basis of $g$, and set

$$
\mathscr{B}_{\infty ; \alpha, q}=\bigcap_{n \geqq 1} \mathscr{B}_{n ; \alpha, q} .
$$

Since the $V_{\alpha, q}$ are restrictions of the $V(x)$ and since $\mathscr{B}_{n ; \alpha, q} \subseteq \mathscr{B}_{n}$, it immediately follows that

$$
\begin{array}{ll}
V_{\alpha, q}(x+y) a=V_{\alpha, q}(x) a+V_{\alpha, q}(y) a, & x, y \in g, a \in \mathscr{B}_{1 ; \alpha, q}, \\
\left(\operatorname{ad} V_{\alpha, q}(x)\right)\left(V_{\alpha, q}(y)\right) a=V_{\alpha, q}((\operatorname{ad} x)(y)) a, & \mathrm{x}, \mathrm{y} \in g, a \in \mathscr{B}_{2 ; \alpha, q} .
\end{array}
$$

Thus the family $V_{\alpha, q}=\left\{V_{\alpha, q}(x) ; x \in g\right\}$ on $\mathscr{B}_{\alpha, q}^{S}$ satisfies the properties (2.1) of a representation of $g$ except for the density of $\mathscr{B}_{\infty ; \alpha, q}$ in $\mathscr{B}_{\alpha, q}^{S}$. It is our aim to establish this density property from the estimates of Theorem 4.1 whenever $q<\infty$.

First remark that the $\mathscr{B}_{n ; \alpha, q}$ are Banach spaces with respect to the norms

$$
a \rightarrow\|a\|_{n ; \alpha, q}=\|a\|+\sup _{1 \leqq m \leqq n} \varrho_{m ; \alpha, q}(a),
$$

where the seminorms $\varrho_{m ; \alpha, q}$ are defined by

$$
\varrho_{m ; \alpha, q}(a)=\sup _{1 \leqq i_{1}, \ldots, i_{m} \leqq d}\left\|V_{\alpha, q}\left(x_{i_{1}}\right) \ldots V_{\alpha, q}\left(x_{i_{m}}\right) a\right\|_{\alpha, q}^{S} .
$$

Second note that the restriction of $S$ to $\mathscr{B}_{\alpha, q}^{S}$ is a semigroup which satisfies the same bounds as $S$ on $\mathscr{B}$, i.e.,

$$
\left\|S_{t} a\right\|_{\alpha, q}^{S} \leqq N e^{\varrho t}\|a\|_{\alpha, q}^{S}
$$

for all $a \in \mathscr{B}_{\alpha, q}^{S}$ and $t \geqq 0$. Moreover, if $q<\infty$ the restriction is $\|\cdot\|_{\alpha, q}^{S}$-continuous, by the Lebesgue dominated convergence theorem. Hence the generator of the restriction $\Delta_{\alpha, q}$ is well-defined as a norm-closed, norm-densely defined, operator on $\mathscr{B}_{\alpha, q}^{S}$ and in fact $\Delta_{\alpha, q}$ is the restriction of $\hat{\Delta}$ to the domain

$$
D\left(\Delta_{\alpha, q}\right)=\left\{a \in D(\hat{\Delta}) ; \hat{\Delta} a \in \mathscr{B}_{\alpha, q}^{S}\right\} .
$$

Next $\Delta_{\alpha, q}+\varrho I$ generates a semigroup on $\mathscr{B}_{\alpha, q}^{S}$ which is uniformly bounded and consequently $\nabla_{\alpha, q}=\left(\Delta_{\alpha, q}+\varrho I\right)^{1 / 2}$ is also well-defined. This latter operator generates the Poisson semigroup associated with the restriction of $S$ to $\mathscr{B}_{\alpha, q}^{S}$ or, equivalently, the restriction to $\mathscr{B}_{\alpha, q}^{S}$ of the Poisson semigroup associated with $S$ on $\mathscr{B}$. Alternatively $\nabla_{\alpha, q}$ can be identified as the restriction of $\nabla=(\hat{\Delta}+\varrho I)^{1 / 2}$ to the domain

$$
D\left(\nabla_{\alpha, q}\right)=\left\{a \in D(\nabla) ; \nabla a \in \mathscr{B}_{\alpha, q}^{S}\right\}
$$


Note that since the Poisson semigroup associated with $S$ is uniformly bounded with bound $N$ one has the dissipativity estimates

$$
\left\|\left(I+\nabla_{\alpha, q}\right)^{m} a\right\|_{\alpha, q}^{S} \geqq N^{-1}\|a\|_{\alpha, q}^{S} \geqq N^{-1}\|a\|
$$

for all $a \in D\left(\nabla_{\alpha, q}^{m}\right)$, and $m=1,2, \ldots$ Moreover, the subspaces $D\left(\nabla_{\alpha, q}^{m}\right)$ of $\mathscr{B}_{\alpha, q}^{S}$ are all norm-dense in $\mathscr{B}_{\alpha, q}^{S}$ and are Banach spaces with respect to the equivalent norms

$$
\begin{aligned}
& a \rightarrow\left\|\left(I+\nabla_{\alpha, q}\right)^{m} a\right\|_{\alpha, q}^{S}, \\
& a \rightarrow \sup _{0 \leqq p \leqq m}\left\|\left(\nabla_{\alpha, q}\right)^{p} a\right\|_{\alpha, q}^{S} .
\end{aligned}
$$

If $m=2 n$ is even then $\nabla_{\alpha, q}^{2 n}=\Delta_{\alpha, q}^{n}$ and one also has the equivalent norms

$$
\begin{aligned}
& a \rightarrow\left\|\left((1+\varrho) I+\Delta_{\alpha, q}\right)^{n} a\right\|_{\alpha, q}^{S}, \\
& a \rightarrow \sup _{0 \leqq p \leqq n}\left\|\left(\Delta_{\alpha, q}\right)^{p} a\right\|_{\alpha, q}^{S} .
\end{aligned}
$$

Now density of the $\mathscr{B}_{n ; \alpha, q}$ follows by comparison with the $D\left(\nabla_{\alpha, q}^{n}\right)$.

Theorem 5.1. Assume $S$ satisfies Conditions $B_{1}$ and $S_{4}$, and that $\alpha \in\langle 0,1 / 2\rangle$, $q \in[1, \infty\rangle$. Then

and

$$
\mathscr{B}_{2 n ; \alpha, q}=D\left(\nabla_{\alpha, q}^{2 n}\right)=D\left(\Delta_{\alpha, q}^{n}\right)
$$

$$
\mathscr{B}_{2 n-1 ; \alpha, q} \supseteq D\left(\nabla_{\alpha, q}^{2 n-1}\right)
$$

for all $n=1,2, \ldots$ In particular the subspaces $\mathscr{B}_{n ; \alpha, q}$ are $\|\cdot\|_{\alpha, q^{-}}^{S}$ dense in $\mathscr{B}_{\alpha, q}^{S}$.

Proof. Throughout the proof we will work on $\mathscr{B}_{\alpha, q}^{S}$ and for notational simplicity we omit the suffices $\alpha, q$ on the $V, \nabla$, and $\Delta$. Thus we identify $V(x)$ and $V_{\alpha, q}(x), \nabla$ and $\nabla_{\alpha, q}$, and $\Delta$ will now denote the closed Laplacian on $\mathscr{B}_{\alpha, q}^{S}$ which generates the restriction of $S$.

We begin by proving (5.2) and (5.3) for $n=1$.

The estimates (4.1) and (4.2) establish that

and

$$
\left\|V\left(x_{i}\right) a\right\|_{\alpha, q}^{S} \leqq k_{2 \alpha, q}\|(I+\nabla) a\|_{\alpha, q}^{S}
$$

$$
\left\|V\left(x_{i}\right) V\left(x_{j}\right) a\right\|_{\alpha, q}^{S} \leqq \ell_{2 \alpha, q}\|(I+(\Delta+\varrho I)) a\|_{\alpha, q}^{S}
$$

for all $a \in \mathscr{B}_{4}$ and $i, j=1, \ldots, d$, where $x_{1}, \ldots, x_{d}$ is an arbitrary of $g$. Now if $a \in D(\nabla)$ then $S_{t} a \in \mathscr{B}_{4}$ and $\left\|S_{t} a-a\right\|_{\alpha, q}^{S} \rightarrow 0,\left\|\nabla S_{t} a-\nabla a\right\|_{\alpha, q}^{S} \rightarrow 0$, as $t \rightarrow 0$, because $S$ is $\|\cdot\| \|_{\alpha, q^{-}}^{S}$ continuous. Therefore (5.4) extends to all $a \in D(\nabla)$ by continuity and this together with (5.1) establishes that

$$
D(\nabla) \subseteq \mathscr{B}_{1 ; \alpha, q} .
$$

Similarly if $a \in D(\Delta)$ then $\left\|S_{t} a-a\right\|_{\alpha, q}^{S} \rightarrow 0,\left\|\Delta S_{t} a-\Delta a\right\|_{\alpha, q}^{S} \rightarrow 0$, as $t \rightarrow 0$. But it also follows from (5.4) that $\left\|V\left(x_{i}\right) S_{t} a-\mathrm{V}\left(x_{i}\right) a\right\|_{\alpha, q}^{S} \rightarrow 0$ as $t \rightarrow 0$. Therefore (5.5) extends to all $a \in D(\Delta)$. But by general semigroup theory one has dissipativity estimates

$$
\|(I+(\Delta+\varrho I)) a\|_{\alpha, q}^{S} \geqq N^{-1}\|a\|_{\alpha, q}^{S} \geqq N^{-1}\|a\|
$$


and relative bounds

$$
\|(I+\nabla) a\|_{\alpha, q}^{S} \leqq k_{\alpha, q}^{\prime}\|(I+(\Delta+\varrho I)) a\|_{\alpha, q}^{S}
$$

for all $a \in D(\Delta)$. Combination of these estimates establishes that $\|\cdot\|_{2 ; \alpha, q}$ is dominated by the norm $a \in D(\Delta) \rightarrow\|(I+(\Delta+\varrho I)) a\|_{\alpha, q}^{S}$ and hence

Conversely (4.3) states that

$$
D(\Delta) \subseteq \mathscr{B}_{2: \alpha, q}
$$

$$
\|\Delta a\|_{\alpha, q}^{S} \leqq d\|a\|_{2 ; \alpha, q}
$$

for all $a \in \mathscr{B}_{2 ; \alpha, q}$ and it follows from this estimate and (5.7) that

$$
\mathscr{B}_{2 ; \alpha, q} \subseteq D(\Delta) \text {. }
$$

This result combined with the previous inclusion establishes (5.2) with $n=1$.

In order to establish (5.2) and (5.3) for higher $n$ we use the structure relations of $g$ and the property $S_{t} \mathscr{B} \subseteq \mathscr{B}_{4} \subseteq \mathscr{B}_{3}$ to deduce that

$$
(\operatorname{ad} \Delta)(V(x)) S_{t} a=P_{2}(V) S_{t} a
$$

for all $a \in \mathscr{B}$ where $P_{2}(V)$ is a second order polynomial in the $V\left(x_{i}\right)$. In particular

$$
\Delta V(x) S_{t} a=V(x) S_{t} \Delta a+P_{2}(V) S_{t} a
$$

for all $a \in D(\Delta)$. If, however, $a \in D\left(\nabla^{3}\right) \subseteq D(\Delta)$ then $\left\|S_{t} a-a\right\|_{\alpha, q}^{S} \rightarrow 0$, $\left\|S_{t} \Delta a-\Delta a\right\|_{\alpha, q}^{S} \rightarrow 0$, and in addition it follows from (5.4) and (5.5) that $\left\|V(x) S_{t} a-V(x) a\right\|_{\alpha, q}^{S} \rightarrow 0, \quad\left\|V(x) S_{t} \Delta a-V(x) \Delta a\right\|_{\alpha, q}^{S} \rightarrow 0, \quad$ and $\| P_{2}(V) S_{t} a$ $-P_{2}(V) a \|_{\alpha, q}^{S} \rightarrow 0$, as $t \rightarrow 0$. Therefore one concludes from (5.8) by continuity that $a \in D(\Delta V(x))$. Thus $D\left(\nabla^{3}\right) \subseteq D(\Delta V(x))$ and also

$$
V(x) D\left(\nabla^{3}\right) \subseteq D(\Delta)=\mathscr{B}_{2 ; \alpha, q} .
$$

Consequently $D\left(\nabla^{3}\right)$ is a subspace of $\mathscr{B}_{2 ; \alpha, q}$. But it then follows from (5.4), (5.5), and the structure relations of $g$ that

$$
\left\|V\left(x_{i}\right) V\left(x_{j}\right) V\left(x_{k}\right) a\right\|_{\alpha, q}^{S} \leqq k_{\alpha, q}^{\prime}\left(\|a\|_{\alpha, q}^{S}+\sup _{1 \leqq m \leqq 3}\left\|\nabla^{m} a\right\|_{\alpha, q}^{S}\right)
$$

for all $a \in D\left(\nabla^{3}\right)$, all $i, j, k=1, \ldots, d$, and a suitable $k_{\alpha, q}^{\prime}$. In combination with the previous estimates this gives

$$
D\left(\nabla^{3}\right) \subseteq \mathscr{B}_{3 ; \alpha, q}
$$

Next since $S_{t} \mathscr{B} \subseteq \mathscr{B}_{4}$ it follows from another application of the structure relations of $g$ that

$$
\Delta V(x) V(y) S_{t} a=V(x) V(y) S_{t} \Delta a+P_{3}(V) S_{t} a
$$

for $a \in D(\Delta)$ and $x, y \in g$, where $P_{3}(V)$ is a third order polynomial in the $V(x)$. Consequently arguing as above one deduces from (5.4), (5.5), and (5.9), that 
$V(x) V(y) D\left(\Delta^{2}\right) \subseteq D(\Delta)$ for all $x, y \in g$ and in addition one has an estimate

$$
\left\|V\left(x_{i_{1}}\right) \ldots V\left(x_{i_{4}}\right) a\right\|_{\alpha, q}^{S} \leqq k_{\alpha, q}^{\prime \prime}\left(\|a\|_{\alpha, q}^{S}+\sup _{1 \leqq m \leqq 4}\left\|\nabla^{m} a\right\|_{\alpha, q}^{S}\right)
$$

for all $a \in D\left(\Delta^{2}\right)$. In combination with the earlier estimates this gives

$$
D\left(\Delta^{2}\right)=D\left(\nabla^{4}\right) \subseteq \mathscr{B}_{4 ; \alpha, q} .
$$

Combining these conclusions one has

$$
\mathscr{B}_{4 ; \alpha, q}=D\left(\nabla^{4}\right)=D\left(\Delta^{2}\right)
$$

and this establishes (5.2) and (5.3) for $n=1,2$. The proof for higher values of $n$ then proceeds by induction.

Let us assume (5.2) and (5.3) are true for $n=1,2, \ldots, N$ where $N \geqq 2$. Next set $R$ $=(I+(\Delta+\varrho I))^{-1}$ and $S=(I+\nabla)^{-1}$. Then $D\left(\nabla^{2 N+1}\right)=R^{N} S \mathscr{B} \subseteq D(\nabla)$ and

$$
\begin{aligned}
V(x) R^{N} S a & =R^{N} V(x) S a-\left(\operatorname{ad} R^{N}\right)(V(x)) S a \\
& =R^{N} V(x) S a+\sum_{n=1}^{N}\left(\begin{array}{l}
N \\
n
\end{array}\right) R^{N-n}(\operatorname{ad} R)^{n}(V(x)) S a .
\end{aligned}
$$

But

$$
(\operatorname{ad} R)(V(x)) S a=-R(\operatorname{ad} \Delta)(V(x)) R S a
$$

for all $a \in \mathscr{B}$, because $D\left(\nabla^{3}\right)=R S \mathscr{B}=\mathscr{B}_{3 ; \alpha, q}$. Moreover

$$
(\operatorname{ad} \nabla)(V(x)) a=P_{2}(V) a
$$

for all $a \in \mathscr{B}_{3 ; \alpha, q}$, where $P_{2}(V)$ is a second-order polynomial in the $V(x)$. Therefore

$$
(\operatorname{ad} R)(V(x)) S a=R P_{2}(V) R S a
$$

for all $a \in \mathscr{B}$. But if one then successively defines polynomials $P_{m}(V)$ of order $m$ much that

$$
(\operatorname{ad} \Delta)\left(P_{m}(V)\right) a=P_{m+1}(V) a
$$

for all $a \in \mathscr{B}_{m+2 ; \alpha, q}$ and $m=2, \ldots, N$ then iteration of this argument establishes that

$$
(\operatorname{ad} R)^{n}(V(x)) S a=(-1)^{n} R^{n} P_{n+1}(V) R^{n} S a
$$

for $a \in \mathscr{B}$ and $n=2, \ldots, N$ because

$$
R^{n} S \mathscr{B}=D\left(\nabla^{2 n+1}\right) \subseteq D\left(\Delta^{2 n}\right)=\mathscr{B}_{2 n ; \alpha, q} \subseteq \mathscr{B}_{n+2 ; \alpha, q} .
$$

The equality $D\left(\Delta^{n}\right)=\mathscr{B}_{2 n ; \alpha, q}$ follows from the induction hypothesis and the inclusion $\mathscr{B}_{2 n ; \alpha, q} \subseteq \mathscr{B}_{n+2 ; \alpha, q}$ follows because $n \geqq 2$. But combining (5.10), (5.11), and (5.12), gives

$$
V(x) R^{N} S a=R^{N}\left(V(x)+\sum_{n=1}^{N}(-1)^{n}\left(\begin{array}{l}
N \\
n
\end{array}\right) P_{n+1}(V) R^{n}\right) S a
$$

for all $a \in \mathscr{B}$. In particular one concludes that $V(x) D\left(\nabla^{2 N+1}\right) \subseteq D\left(\nabla^{2 N}\right)$. But $D\left(\nabla^{2 N}\right)=\mathscr{B}_{2 N ; \alpha, q}$ by the induction hypothesis and hence $D\left(\nabla^{2 N+1}\right)$ is in the domain of all monomials of order $2 N+1$ in the $V(x)$. Therefore, using the induction 
hypothesis and the structure relations of $g$, one estimates that if $a \in D\left(\nabla^{2 N+1}\right)$ then

$$
\begin{aligned}
\left\|\left(\prod_{j=1}^{2 N} V\left(x_{i_{j}}\right)\right) V(x) a\right\|_{\alpha, q}^{S} & \leqq k \sup _{0 \leqq p \leqq N}\left\|\Delta^{p} V(x) a\right\|_{\alpha, q}^{S} \\
\leqq & k^{\prime}\left(\sup _{0 \leqq p \leqq 2 N+1}\left\|\nabla^{p} a\right\|_{\alpha, q}^{S}\right. \\
& \left.+\sup _{0 \leqq p \leqq N}\left\|\left(\operatorname{ad} \Delta^{p}\right)(V(x)) a\right\|_{\alpha, q}^{S}\right) .
\end{aligned}
$$

But $\left(\operatorname{ad} \Delta^{p}\right)(V(x))$ is a polynomial of order $2 p$ in the $V\left(x_{i}\right)$ and since $2 p \leqq 2 N$ one has

$$
\left\|\left(\prod_{j=1}^{2 N} V\left(x_{i_{j}}\right)\right) V(x) a\right\|_{\alpha, q}^{S} \leqq k^{\prime \prime} \sup _{0 \leqq p \leqq 2 N+1}\left\|\nabla^{p} a\right\|_{\alpha, q}^{S}
$$

by another use of the induction hypothesis. This establishes that

$$
D\left(\nabla^{2 N+1}\right) \subseteq \mathscr{B}_{2 N+1 ; \alpha, q} .
$$

Next a very similar calculation gives

$$
V(x) V(y) R^{N+1} a=R^{N}\left(V(x) V(y)+\sum_{n=1}^{N}(-1)^{n}\left(\begin{array}{l}
N \\
n
\end{array}\right) Q_{n+1}(V) R^{n}\right) R a
$$

for all $a \in \mathscr{B}$, where the polynomials $Q$ are now defined successively by

$$
(\operatorname{ad} \Delta)(V(x) V(y)) a=Q_{3}(V) a
$$

for $a \in \mathscr{B}_{4 ; \alpha, q}$ and

$$
(\operatorname{ad} \Delta)\left(Q_{m}(V)\right) a=Q_{m+1}(V) a
$$

for all $a \in \mathscr{B}_{m+2 ; \alpha, q}$ and $m=3, \ldots, N$. In order to justify this calculation it is sufficient that $D\left(\Delta^{n+1}\right) \subseteq \mathscr{B}_{n+3 ; \alpha, q}$ for all $n \leqq N$. But $D\left(\nabla^{2 n+1}\right) \subseteq D\left(\nabla^{2 n+1}\right) \subseteq \mathscr{B}_{2 n+1 ; \alpha, q}$ by the induction hypothesis if $n<N$ and by the previous argument if $n=N$. Therefore one deduces that $V(x) V(y) D\left(\Delta^{N+1}\right) \subseteq D\left(\Delta^{N}\right)$, and hence $D\left(\Delta^{N+1}\right)$ is in the domain of all monomials of order $2(N+1)$ in the $V(x)$. Then if $a \in D\left(\Delta^{N+1}\right)$ one estimates that

$$
\begin{array}{rl}
\left\|\left(\prod_{j=1}^{N} V\left(x_{i_{j}}\right)\right) V(x) V(y) a\right\|_{\alpha, q}^{S} & k \sup _{0 \leqq p \leqq N}\left\|\Delta^{p} V(x) V(y) a\right\|_{\alpha, q}^{S} \\
\leqq & k^{\prime}\left(\sup _{0 \leqq p \leqq N+1}\left\|\Delta^{p} a\right\|_{\alpha, q}^{S}\right. \\
& \left.+\sup _{0 \leqq p \leqq N}\left\|\left(\operatorname{ad} \Delta^{p}\right)(V(x) V(y)) a\right\|_{\alpha, q}^{S}\right)
\end{array}
$$

by the induction hypothesis. Since $\left(\operatorname{ad} \Delta^{p}\right)(V(x) V(y))$ is a polynomial of order $2 p+1$ in the $V\left(x_{i}\right)$, and $p \leqq N$, it then follows from the earlier calculation that

Therefore

$$
\left\|\left(\prod_{j=1}^{N} V\left(x_{i_{j}}\right)\right) V(x) V(y) a\right\|_{\alpha, q}^{S} \leqq k^{\prime \prime} \sup _{0 \leqq p \leqq 2 N}\left\|\nabla^{p} a\right\|_{\alpha, q}^{S} .
$$

$$
D\left(\Delta^{N+1}\right) \subseteq \mathscr{B}_{2 N+2 ; \alpha, q} .
$$


Conversely if $a \in \mathscr{B}_{2 N+2 ; \alpha, q}$ then

$$
\left\|\Delta^{N+1} a\right\|_{\alpha, q}^{S} \leqq \sum_{i_{1}=1}^{d} \ldots \sum_{i_{n+1}=1}^{d}\left\|\left(\prod_{j=1}^{N+1} V\left(x_{i_{j}}\right)^{2}\right) a\right\|_{\alpha, q}^{S},
$$

and consequently

$$
\mathscr{B}_{2 N+2 ; \alpha, q} \subseteq D\left(\Delta^{N+1}\right) .
$$

Combining these conclusions one deduces that (5.2) and (5.3) are now valid for $n=N+1$. Therefore (5.2) and (5.3) are valid for all $n \geqq 1$ by induction.

It is unclear whether the hypotheses of Theorem 5.1 are sufficient to ensure that $\mathscr{B}_{2 n-1 ; \alpha, q}=D\left(\nabla_{\alpha, q}^{2 n-1}\right)$. Theorem 4.5 gives a result in this direction,

$$
\mathscr{B}_{1 ; \alpha, q}^{S}=D\left(\nabla_{\alpha, q}\right)
$$

but the proof requires $\|\cdot\|_{1}$-continuity of $S$ restricted to $\mathscr{B}_{1}$ in addition to Conditions $B_{1}$ and $S_{3}$. Moreover, the spaces $\mathscr{B}_{1 ; \alpha, q}^{S}$ defined by (4.16) and (4.17) differ in a significant manner from the spaces $\mathscr{B}_{1 ; \alpha, q}$. But this motivates the comparison of $D\left(\nabla_{\alpha, q}^{n}\right)$ with the higher order analogues of the $\mathscr{B}_{1 ; \alpha, q}^{S}$, i.e. with the spaces

$$
\mathscr{B}_{n ; \alpha, q}^{S}=\left\{a \in \mathscr{B}_{n} ; \int_{0}^{1} d \mu(t)\left(t^{-\alpha}\left\|\left(I-S_{t}\right) a\right\|_{n}\right)^{q}<\infty\right\}
$$

equipped with the norms

$$
\|a\|_{n ; \alpha, q}^{S}=\|a\|_{n}+\left(\int_{0}^{1} d \mu(t)\left(t^{-\alpha}\left\|\left(I-S_{t}\right) a\right\|_{n}\right)^{q}\right)^{1 / q}
$$

If $S$ leaves $\mathscr{B}_{n}$ invariant and is $\|\cdot\|_{n}$-continuous on $\mathscr{B}_{n}$ then these spaces correspond to the Lipschitz spaces of $S$ restricted to $\mathscr{B}_{n}$. If on the other hand $S$ does not leave $\mathscr{B}_{n}$ invariant these spaces could be quite sparse and this indicates that strong smoothness properties of $S$ would be necessary to prove that $D\left(\nabla_{\alpha, q}^{n}\right) \subseteq \mathscr{B}_{n ; \alpha, q}^{S}$. This is indeed the case. One has

$$
\sup _{0 \leqq m \leqq n}\left\|\Delta^{m} a\right\|_{\alpha, q}^{S} \leqq d^{n}\|a\|_{2 n ; \alpha, q}^{S}
$$

and hence $\mathscr{B}_{2 n ; \alpha, q}^{S} \subseteq D\left(\Delta_{\alpha, q}^{n}\right)$ with no smoothness assumptions but the converse inclusion is more delicate. If, for example, one has $S_{t} \mathscr{B} \subseteq \mathscr{B}_{2}$ and $S$ is $\|\cdot\|_{2}$-continuous on $\mathscr{B}_{2}$ then

$$
\left\|\left(I-S_{t}\right) a\right\|_{2} \leqq \int_{0}^{t} d s\left\|S_{s} \Delta a\right\|_{2}
$$

for all $a \in \mathscr{B}_{2}$. Consequently

$$
\begin{aligned}
\|a\|_{2 ; \alpha, q}^{S} & \leqq\|a\|_{2}+\alpha^{-1}\left(\int_{0}^{1} d \mu(t)\left(t^{1-\alpha}\left\|S_{t} \Delta a\right\|_{2}\right)^{q}\right)^{1 / q} \\
& \leqq\|a\|_{2 ; \alpha, q}+\alpha^{-1}\|\Delta a\|_{\alpha, q ; 2}
\end{aligned}
$$

and it follows from Theorems 3.5 and 5.1 that

$$
\|a\|_{2 ; \alpha, q}^{S} \leqq k_{\alpha, q}\left(\|a\|_{\alpha, q}+\|\Delta a\|_{\alpha, q}\right)
$$


if $S$ also satisfies Conditions $B_{1}$ and $S_{4}$. Thus Conditions $B_{1}, S_{3}$, and $\|\cdot\|_{1}$-continuity of $S$ ensure that

$$
\mathscr{B}_{1 ; \alpha, q}^{S}=D\left(\nabla_{\alpha, q}\right)
$$

and Conditions $\mathscr{B}_{1}, S_{4}$, and $\|\cdot\|_{2}$-continuity of $S$ give

$$
\mathscr{B}_{2 ; \alpha, q}^{S}=D\left(\nabla_{\alpha, q}^{2}\right)=D\left(\nabla_{\alpha, q}\right) \text {. }
$$

These arguments can be generalized to conclude that if $S$ satisfies Conditions $B_{1}$ and $S_{n+2}$, and $S$ restricted to $\mathscr{B}_{n}$ is $\|\cdot\|_{n}$-continuous, then

$$
\mathscr{B}_{m ; \alpha, q}^{S}=D\left(\nabla_{\alpha, q}^{m}\right)
$$

for $m=1,2, \ldots, n$. This is in particular the case if $(\mathscr{B}, g, V)$ is the differential of a continuous group representation because all the smoothness and continuity conditions on $S$ are satisfied for all $n$.

\section{Smoothness and Integrability}

Finally we can use the foregoing analysis to analyze smoothness properties of the heat semigroup and criteria for integrability of the representation of $g$. In particular we improve some of the recent results on these topics [BGJR].

Theorem 6.1. Assume $S$ satisfies Conditions $B_{1}$ and $S_{4}$.

It follows that $S$ satisfies Conditions $B_{n}$ and $S_{n}$ for all $n \geqq 1$.

Proof. First it follows from Proposition 3.1 that $S$ satisfies $B_{2}$ and $B_{3}$. But Condition $B_{2}$ implies that $S$ is holomorphic, i.e. there is a $c>0$ such that $\left\|\Delta S_{t}\right\| \leqq c t^{-1}$ for all $t \in\langle 0,1\rangle$. This, however, implies that the restrictions of $S$ to the Lipschitz spaces $\mathscr{B}_{\alpha, q}^{S}, q<\infty$, are also holomorphic. The restrictions are $\|\cdot\| \|_{\alpha, q}^{S}$-continuous because $q<\infty$, by the Lebesgue dominated convergence theorem, but in addition one has the estimates

$$
\begin{aligned}
\left\|\Delta S_{t} a\right\|_{\alpha, q}^{S} & =\left\|\Delta S_{t} a\right\|+\left(\int_{0}^{1} d \mu(s)\left(s^{-\alpha}\left\|\Delta S_{t}\left(I-S_{s}\right) a\right\|\right)^{q}\right)^{1 / q} \\
& \leqq C\|a\|_{\alpha, q}^{S} t^{-1}
\end{aligned}
$$

for all $t \in\langle 0,1\rangle$ and this is equivalent to holomorphy.

Second let $\Delta_{\alpha, q}$ denote the generator of $S$ restricted to $\mathscr{B}_{\alpha, q}^{S}$. It follows from the holomorphy property that

$$
S_{t} \mathscr{B}_{\alpha, q}^{S} \subseteq D\left(\Delta_{\alpha, q}^{n}\right)
$$

for all $n \geqq 1$ and $t>0$. But if $\alpha \in\langle 0,1 / 2\rangle$ then Theorem 5.1 gives the identification

Therefore

$$
D\left(\Delta_{\alpha, q}^{n}\right)=\mathscr{B}_{2 n ; \alpha, q} \text {. }
$$

for all $n \geqq 1$ and $t>0$.

$$
S_{t} \mathscr{B}_{\alpha, q}^{S} \subseteq \mathscr{B}_{2 n ; \alpha, q}
$$

Finally one has

$$
S_{t / 2} \mathscr{B} \subseteq \mathscr{B}_{4} \subseteq \mathscr{B}_{\alpha, q}
$$


for all $t>0$ by Condition $S_{4}$ and hence

$$
S_{t} \mathscr{B} \subseteq S_{t / 2} \mathscr{B}_{\alpha, q} \subseteq \mathscr{B}_{2 n ; \alpha, q} \subseteq \mathscr{B}_{2 n} .
$$

Therefore $S$ satisfies Condition $S_{n}$ for all $n \geqq 1$ and, by Proposition 3.1, it also satisfies Condition $B_{n}$ for all $n \geqq 1$.

If Theorem 6.1 is combined with Theorem 2.1 and Corollary 2.6 of [BGJR] one obtains an even more striking conclusion.

Corollary 6.2. Assume $S$ satisfies Conditions $B_{1}$ and $S_{4}$, then $S_{t} \mathscr{B} \subseteq \mathscr{B}_{\infty}$ for all $t>0$ and there exist $k, \ell>0$ such that

$$
\sup _{1<i_{1}, \ldots, i_{n} \leqq d}\left\|V\left(x_{i_{1}}\right) \ldots V\left(x_{i_{n}}\right) S_{t} a\right\| \leqq k \ell^{n} n !\|a\| t^{-n / 2}
$$

for all $t \in\langle 0,1\rangle, a \in \mathscr{B}$, and $n \geqq 1$, where $x_{1}, \ldots, x_{d}$ is a basis of $g$. In particular the representation $(\mathscr{B}, g, V)$ has a dense set of analytic elements.

The primary interest of this last conclusion is that it can be used to give criteria which ensure that $(\mathscr{B}, g, V)$ is integrable, i.e. there exists a group representation $(\mathscr{B}, G, U)$ such that $V=d U$. It is necessary for integrability that the $V(x)$ are weakly conservative in the sense that there exists a basis $x_{1}, \ldots, x_{d}$ of $g, \delta, m \in\langle 0,1\rangle$, and $\omega>0$, such that

$$
\left\|\left(I+\varepsilon V\left(x_{i}\right)\right)^{n} a\right\| \geqq m(1-|\varepsilon| \omega)^{n}\|a\|
$$

for all $a \in \mathscr{B}_{n}$, all $n=1,2, \ldots$, all $\varepsilon \in\langle-\delta, \delta\rangle$, and all $i=1, \ldots, d$. Now combination of the previous results with those of [BGJR] and [BBR] gives the following integrability criteria.

Theorem 6.3. Let $(\mathscr{B}, g, V)$ be a representation of the Lie algebra $g$ for which the $V$ are weakly conservative and let $\Delta$ be the Laplacian associated with some basis $x_{1}, \ldots, x_{d}$ of $g$. Then the following conditions are equivalent;

1. a) $\Delta$ has an extension which generates a strongly continuous semigroup $S$,

b) $S_{t} \mathscr{B} \subseteq \mathscr{B}_{4}(V), t>0$,

c) there is a $c>0$ such that

$$
\sup _{1 \leqq i \leqq d}\left\|V\left(x_{i}\right) S_{t} a\right\| \leqq c\|a\| t^{-1 / 2}
$$

for all $a \in \mathscr{B}$ and $t \in\langle 0,1\rangle$,

2. a) 4 has an extension $\hat{\Delta}$ which generates a strongly continuous holomorphic semigroup,

b) $D\left(\hat{\Delta}^{n}\right) \subseteq \mathscr{B}_{4}(V)$ for some $n \geqq 2$,

c) $D(\hat{\Delta}) \subseteq \mathscr{B}_{1}(V)$ and there is a $c^{\prime}>0$ such that

$$
\sup _{1 \leqq i \leqq d}\left\|V\left(x_{i}\right) a\right\| \leqq \varepsilon\|\hat{\Delta} a\|+c^{\prime} \varepsilon^{-1}\|a\|
$$

for all $a \in D(\widehat{\Delta})$ and $\varepsilon \in\langle 0,1\rangle$,

3. $(\mathscr{B}, g, V)$ is integrable.

Proof. The equivalence of Conditions 1 and 3 follows from Theorem 2.8 and Remark 2.9 of [BGJR] together with Theorem 6.1 above once one remarks that Condition $1 \mathrm{~b}$ implies that the generator of $S$ is the closure $\bar{\Delta}$ of $\Delta$. 
Next Condition 1 implies $S$ satisfies Condition $B_{2}$ by Proposition 3.1 and hence $S$ is holomorphic. Moreover Condition 1 implies the estimates of Corollary 6.2 and by Laplace transformation one concludes that $D\left(\hat{\Delta}^{m}\right) \subseteq \mathscr{B}_{n}(V)$, where $m=n / 2+1$ if $n$ is even and $m=(n+1) / 2$ if $n$ is odd. Then the estimate of Condition 2 followis from that of Condition 1c by Laplace transformation as in Lemma 2.2 of [BGJR]. Thus $2 \Rightarrow 1$.

We conclude with a few remarks about the integrability criteria of Theorem 6.3.

First, if the representation $(\mathscr{B}, g, V)$ is integrable then $\Delta$ is closable and its closure $\bar{\Delta}$ generates a continuous semigroup $S$, and hence integrability implies that $\bar{\Delta}$ is the unique generator extension of $\Delta$.

Second, if Conditions $1 \mathrm{a}$ and $1 \mathrm{~b}$ are satisfied then the generator of $S$ is again $\bar{\Delta}$, because $\bigcup_{t>0} S_{t} \mathscr{B} \subseteq D(\Delta)$ by Condition $1 \mathrm{~b}$. Similarly if Conditions $2 \mathrm{a}$ and $2 \mathrm{~b}$ are valid then $\hat{\Delta}=\bar{\Delta}$. This follows because $\bigcup_{t>0} S_{t} \mathscr{B} \subseteq D\left(\hat{\Delta}^{n}\right) \subseteq \mathscr{B}_{4}(V) \subseteq D(\Delta)$, where the first inclusion results from holomorphy of $S$ and the second from Condition $2 \mathrm{~b}$.

Third, Condition 1a does not necessarily imply Conditions $1 \mathrm{~b}$ and $1 \mathrm{c}$ nor does Condition 2a imply Conditions $2 \mathrm{~b}$ and $2 \mathrm{c}$. For example, if $\mathscr{B}=C_{0}(0, \infty)$, the continuous functions over $\langle 0, \infty\rangle$ vanishing at 0 and $\infty$, and if $\delta=d / d x$, with $D(\delta)$ the absolutely continuous functions $f \in \mathscr{B}$ such that $f^{\prime} \in \mathscr{B}$, then $-\delta^{2}$ is closed (see [BBR, Example 3.3]) but it is not a generator. Nevertheless it has many extensions which generate holomorphic semigroups, e.g. the operator $\hat{\Delta}$ with $D(\widehat{\Delta})$ the absolutely continuous $f \in \mathscr{B}$ with absolutely continuous derivative $f^{\prime}$ such that $f^{\prime \prime} \in C_{0}(0, \infty)$. This operator generates the holomorphic semigroup $S$ with action

$$
\left(S_{t} f\right)(x)=\int_{0}^{\infty} d y\left(p_{t}(x-y)-p_{t}(x+y)\right) f(y),
$$

where $p_{t}(x)=(4 \pi t)^{-1 / 2} \exp \left\{-x^{2} / 4 t\right\}$ but $D\left(\delta^{2}\right) \subseteq D(\widehat{\Delta}) \nsubseteq D(\delta)$.

Fourth, if the $V\left(x_{i}\right)$ are conservative, i.e. if

$$
\left\|\left(I+\varepsilon V\left(x_{i}\right)\right) a\right\| \geqq\|a\|
$$

for all $a \in \mathscr{B}_{1}, i=1, \ldots, d$, and $\varepsilon \in\langle-\delta, \delta\rangle$, where $\delta>0$, then $\Delta$ is dissipative (see [Rob2, Lemma 3.3]) and hence closable. If the closure $\bar{\Delta}$ of $\Delta$ generates a holomorphic semigroup $S$ then it is conceivable that Conditions $2 \mathrm{~b}$ and $2 \mathrm{c}$ follow automatically for $\bar{\Delta}$. In particular this is the situation of $d=1$. In this special case there is a unique closed operator $V, \Delta=-V^{2}$, and

$$
\|V a\| \leqq \varepsilon\left\|V^{2} a\right\|+2 \varepsilon^{-1}\|a\|
$$

for all $a \in D\left(V^{2}\right)$ and $\varepsilon>0$ by Lemma 2.3 of [Rob2]. It follows that $\Delta=\bar{\Delta}=-V^{2}$, and hence one has

$$
D\left(\bar{\Delta}^{n}\right)=\mathscr{B}_{2 n}(V) .
$$

But one cannot expect (6.1) to be valid if $d>1$. For example, this identification is false [Orn] for the Lie algebra obtained by differentiating the group $G=\mathbb{R}^{d}$ acting as translations on the Banach space $L_{1}\left(\mathbb{R}^{d}\right)$ whenever $d>1$. Nevertheless since this example comes from a group representation, each Laplacian $\Delta$ is closable, $\bar{\Delta}$ 
generates a holomorphic semigroup,

$$
\mathscr{B}_{2 n}(V) \subseteq D\left(\bar{\Delta}^{n}\right) \subseteq \mathscr{B}_{2 n-1}(V)
$$

for each $n \geqq 1$, and there is a $c>0$ such that

$$
\left\|V\left(x_{i}\right) a\right\| \leqq \varepsilon\|\bar{\Delta} a\|+c \varepsilon^{-1}\|a\|
$$

for all $a \in D(\bar{\Delta}), i=1, \ldots, d$, and $\varepsilon \in\langle 0,1\rangle$. The last statements follow from the estimates of [BGJR].

Finally we remark that stronger results are to be expected if $\mathscr{B}$ is a $C^{*}$-algebra and the $V\left(x_{i}\right)$ are $*$-derivations. For example, it has then been established [BBR, $\mathrm{BrJ}$ ] that the $V\left(x_{i}\right)$ are conservative if, and only if, the Laplacian $\Delta$ is dissipative and it is feasible that the representation is integrable if, and only if, $\bar{\Delta}$ generates a holomorphic contraction semigroup. One possible approach to this problem is to prove the validity of Conditions $2 \mathrm{~b}$ and $2 \mathrm{c}$ of Theorem 6.3 for $\bar{\Delta}$.

\section{References}

[BBR] Batty, C.J.K., Bratteli, O., Robinson, D.W.: The heat semigroup, derivations and Reynold's identity. In: Operator algebras and applications. Evans, D.E., Takesaki, M. (eds.). Lond. Math. Soc. Lect. Notes, Vol. 136. Cambridge: Cambridge University Press

[BcF] Berg, C., Forst, G.: Potential theory on locally compact abelian groups. Berlin, Heidelberg, New York: Springer 1975

[BGJR] Bratteli, O., Goodman, F., Jørgensen, P.E.T., Robinson, D.W.: The heat semigroup and integrability of Lie algebras. J. Funct. Anal. 79, 351-397 (1988)

[BrJ] Bratteli, O., Jørgensen, P.E.T.: Conservative derivations and dissipative Laplacians. J. Funct. Anal. 82, 404-441 (1989)

[Bu B] Butzer, P.L., Berens, H.: Semigroups of operators and approximation. Berlin, Heidelberg, New York: Springer 1967

[Orn] Ornstein, D.: A non-inequality for differential operators in the $L_{1}$-norm. Arch. Rat. Mech. Anal. 11, 40-49 (1962)

[Rob1] Robinson, D.W.: Lie groups and Lipschitz spaces. Duke Math. J. 57, 357-395 (1988)

[Rob2] Robinson, D.W.: The differential and integral structure of representations of Lie groups. J. Op. Theor. 19, 95-128 (1988)

[Tri] Triebel, H.: Interpolation theory, function spaces, differential operators. Amsterdam: North-Holland 1978

[Yos] Yosida, K.: Functional analysis. Berlin, Heidelberg, New York: Springer 1968

Communicated by A. Jaffe

Received April 5, 1990 
